\title{
Poisson point processes attached to symmetric diffusions
}

\author{
Masatoshi Fukushima ${ }^{\mathrm{a}, *}$, Hiroshi Tanaka ${ }^{\mathrm{b}}$ \\ ${ }^{a}$ Department of Mathematics, Faculty of Engineering, Kansai University, Suita 564-8680, Japan \\ b 1-4-17-104 Miyamaedaira, Miyamae-ku, Kawasaki 216-0006, Japan
}

Received 31 January 2004; received in revised form 26 September 2004; accepted 1 October 2004

Available online 29 March 2005

\begin{abstract}
Let $a$ be a non-isolated point of a topological space $S$ and $X^{0}=\left(X_{t}^{0}, 0 \leqslant t<\zeta^{0}, P_{x}^{0}\right)$ be a symmetric diffusion on $S_{0}=$ $S \backslash\{a\}$ such that $P_{x}^{0}\left(\zeta^{0}<\infty, X_{\zeta^{0}-}^{0}=a\right)>0, x \in S_{0}$. By making use of Poisson point processes taking values in the spaces of excursions around $a$ whose characteristic measures are uniquely determined by $X^{0}$, we construct a symmetric diffusion $\tilde{X}$ on $S$ with no killing inside $S$ which extends $X^{0}$ on $S_{0}$. We also prove that such a process $\widetilde{X}$ is unique in law and its resolvent and Dirichlet form admit explicit expressions in terms of $X^{0}$.

○) 2005 Elsevier SAS. All rights reserved.
\end{abstract}

\section{Résumé}

Etant donné un point $a$ non isolé d'un espace topologique $S$, nous considérons une diffusion symétrique $X^{0}=\left(X_{t}^{0}, P_{x}^{0}\right)$ dans $S_{0}=S \backslash\{a\}$ telle que $P_{x}^{0}\left(\zeta^{0}<\infty, X_{\zeta^{0}-}^{0}=a\right)>0$ et $P_{x}^{0}\left(\zeta^{0}<\infty, X_{\zeta^{0}-}^{0} \in S_{0}\right)=0$ pour tout $x \in S_{0}$ où $\zeta^{0}$ est la durée de vie. En utilisant les processus de Poisson ponctuels des excursions partant de $a$ dont les mesures caractéristiques sont déterminées par $X^{0}$, nous construirons une diffusion symétrique $\widetilde{X}$ dans $S$ qui est une extension de $X^{0}$ et dont les trajectoires ne disparaisssent pas à l'intérieur de $S$. Nous montrons aussi qu'une telle extension est unique en loi et que sa résolvente et sa forme de Dirichlet admettent les expressions explicites en terme de $X^{0}$.

(c) 2005 Elsevier SAS. All rights reserved.

\footnotetext{
* Corresponding author.

E-mail address: fuku@ipcku.kansai-u.ac.jp (M. Fukushima).
} 


\section{Introduction}

Let $S$ be a locally compact separable metric space and $a$ be a non-isolated point of $S$. We put $S_{0}=S \backslash\{a\}$. The one point compactification of $S$ is denoted by $S_{\Delta}$. When $S$ is compact already, $\Delta$ is added as an isolated point. Let $m$ be a positive Radon measure on $S_{0}$ with $\operatorname{Supp}[m]=S_{0} . m$ is extended to $S$ by setting $m(\{a\})=0$.

We assume that we are given an $m$-symmetric diffusion $X^{0}=\left(X_{t}^{0}, P_{x}^{0}\right)$ on $S_{0}$ with life time $\zeta^{0}$ satisfying the following four conditions:

$$
\text { A.1 } P_{x}^{0}\left(\zeta^{0}<\infty, X_{\zeta^{0}-}^{0} \in\{a\} \cup\{\Delta\}\right)=P_{x}^{0}\left(\zeta^{0}<\infty\right), \quad \forall x \in S_{0}
$$

We define the functions $\varphi(x), u_{\alpha}(x), \alpha>0$, of $x \in S_{0}$ by

$$
\varphi(x)=P_{x}^{0}\left(\zeta^{0}<\infty, X_{\zeta^{0}-}^{0}=a\right), \quad u_{\alpha}(x)=E_{x}^{0}\left(\mathrm{e}^{-\alpha \zeta^{0}} ; X_{\zeta^{0}-}^{0}=a\right) .
$$

A.2 $\varphi(x)>0, \forall x \in S_{0}$.

A.3 $u_{\alpha} \in L^{1}\left(S_{0} ; m\right), \forall \alpha>0$.

A.4 $u_{\alpha} \in C_{b}\left(S_{0}\right), G_{\alpha}^{0}\left(C_{b}\left(S_{0}\right)\right) \subset C_{b}\left(S_{0}\right), \alpha>0$,

where $G_{\alpha}^{0}$ is the resolvent of $X^{0}$ and $C_{b}\left(S_{0}\right)$ is the space of all bounded continuous functions on $S_{0}$.

By making use of excursion-valued Poisson point processes whose characteristic measures are uniquely determined by $X^{0}$, or to be a little more precise, by piecing together those excursions which start from $a$ and return to $a$ and then possibly by adding the last one that never returns to $a$, we shall construct in $\S 4$ of the present paper a process $\widetilde{X}$ on $S$ satisfying

(1) $\underset{\widetilde{X}}{\widetilde{X}}$ is an $m$-symmetric diffusion process on $S$ with no killing inside $S$,

(2) $\widetilde{X}$ is an extension of $X^{0}$ : the process on $S_{0}$ obtained from $\widetilde{X}$ by killing upon the hitting time of $a$ is identical in law with $X^{0}$.

We call a process $\tilde{X}$ on $S$ satisfying (1), (2) a symmetric extension of $X^{0}$.

We shall also prove in $\S 5$ that, under conditions A.1, A.2 for the given $m$-symmetric diffusion $X^{0}$ on $S_{0}$, its symmetric extension is unique in law, satisfies condition A.3 automatically and admits the resolvent expressible as

$$
G_{\alpha} f(x)=G_{\alpha}^{0} f(x)+u_{\alpha}(x) \cdot G_{\alpha} f(a), \quad x \in S_{0}, \quad G_{\alpha} f(a)=\frac{\left(u_{\alpha}, f\right)}{\alpha\left(u_{\alpha}, \varphi\right)+L\left(m_{0}, \psi\right)},
$$

where $(\cdot, \cdot)$ denotes the inner product in $L^{2}\left(S_{0} ; m\right)$ and $L\left(m_{0}, \psi\right)$ is the energy functional in Meyer's sense [21] of the $X^{0}$-excessive measure $m_{0}=\varphi \cdot m$ and $X^{0}$-excessive function $\psi=1-\varphi$.

Furthermore the associated Dirichlet form $(\mathcal{E}, \mathcal{F})$ on $L^{2}(S ; m)$ will be seen in $\S 5$ to have the following simple expression; if we denote by $\mathcal{F}_{e}$ its extended Dirichlet space, then

$$
\begin{aligned}
& \mathcal{F}_{e}=\left\{w=u_{0}+c \varphi: u_{0} \in \mathcal{F}_{0, e}, c \text { constant }\right\}, \quad \mathcal{F}=\mathcal{F}_{e} \cap L^{2}(S ; m), \\
& \mathcal{E}(w, w)=\mathcal{E}\left(u_{0}, u_{0}\right)+c^{2} \mathcal{E}(\varphi, \varphi), \quad \mathcal{E}(\varphi, \varphi)=L\left(m_{0}, \psi\right),
\end{aligned}
$$

where $\left(\mathcal{F}_{0, e}, \mathcal{E}\right)$ is the extended Dirichlet space for the given diffusion $X^{0}$.

In $\S 6$, we shall present four examples. Example 6.1 concerns the uniqueness of the symmetric extension of the one-dimensional absorbing Brownian motion.

Example 6.2 treats the case where $S_{0}$ is a bounded open subset of $\mathbb{R}^{d}(d \geqslant 1), S=S_{0} \cup\{a\}$ is the one point compactification of $S_{0}$ and $X^{0}$ is the absorbing Brownian motion on $S_{0}$. In this case, $\varphi(x)=1, x \in S_{0}$. The resulting Dirichlet form on $L^{2}(S ; m)\left(m\right.$ is the Lebesgue measure on $S_{0}$ extended to $S$ by $\left.m(\{a\})=0\right)$ is given by 


$$
\begin{aligned}
& \mathcal{F}=\left\{w=u_{0}+c: u_{0} \in H_{0}^{1}\left(S_{0}\right), c \text { constant }\right\}, \\
& \mathcal{E}(w, w)=\frac{1}{2} \int_{S_{0}}\left|\nabla u_{0}\right|^{2}(x) \mathrm{d} x,
\end{aligned}
$$

which is easily seen to be regular, strongly local and irreducible recurrent. A more general Dirichlet form of this type will be presented in $§ 3.2$. This type of Dirichlet form first appeared in the paper [8] by the first author and it is recently utilized in a study of the asymptotics of the spectral gap for one parameter family of energy forms [17]. Our study is motivated by a wish to conceive a clearer picture of the sample path of the diffusion on $S$ associated with such a Dirichlet form.

Example 6.3 is essentially one-dimensional, where we shall see that the conditions A.2 and A.3 are satisfied if and only if the boundary is regular in Feller's sense. This example is reminiscent of an example by N. Ikeda and S. Watanabe [14].

Example 6.4 is higher dimensional, where the Dirichlet form associated with the constructed process $\widetilde{X}$ may not be regular.

In order to identify right quantities to describe the excursion-valued Poisson point processes to be constructed in $\S 4$, we shall study in $\S 2$ and $\S 3$ a strongly local regular Dirichlet form on $L^{2}(S ; m)$ for which the point $\{a\}$ has a positive capacity. In particular, we shall find that the Dirichlet form and the associated resolvent admit exactly the above mentioned expressions. Furthermore, we shall see that the entrance law $\left\{\mu_{t}\right\}$ governing the excursion law ought to be determined by

$$
m_{0}=\int_{0}^{\infty} \mu_{t} \mathrm{~d} t
$$

an equation investigated by E.B. Dynkin, R.K. Getoor, P.J. Fitzsimmons and others [11].

In a seminal work [15], K. Itô considered a standard process $X$ on $S$ for which a point $a$ is regular for itself. A Poisson point process $\mathbf{Y}$ taking value in the space of excursions around $a$ was then associated, and it was shown that the stopped process $X^{0}$ obtained from $X$ by the hitting time at $a$ and the characteristic measure of $\mathbf{Y}$ together determine the law of $X$ uniquely. It was implicitly assumed in [15] that the point $a$ is recurrent in the sense that

$$
\varphi(x)=P_{x}\left(\sigma_{a}<\infty\right)=1, \quad x \in S, \quad \sigma_{a}=\inf \left\{t>0: X_{t}=a\right\}
$$

But, as was shown in P.A. Meyer [20], an absorbed Poisson point process can be still associated with $X$ when $\{a\}$ is non-recurrent. See Remark 4.2 in this regard.

Since our present assumption on $X^{0}$ requires $\varphi$ only to be positive, we must handle not only returning excusions from the point $a$ but also non-returning excursions. By restricting ourselves to the case that both $X^{0}$ and $\widetilde{X}$ are symmetric diffusions however, we shall see that the characteristic measures on these different type of excursion spaces are uniquely determined by $X^{0}$ so that, starting with $X^{0}$, we can give an explicit construction of $\tilde{X}$.

The Dirichlet form $(\mathcal{E}, \mathcal{F})$ on $L^{2}(S ; m)$ associated with a symmetric extension $\widetilde{X}$ of $X^{0}$ may not be regular but it is quasi-regular in the sense of [19]. Accordingly we can make use of the quasi-homeomorphism in [3] to connect $\widetilde{X}$ with the regular Dirichlet form studied in $\S 2$, yielding the uniqueness of $\widetilde{X}$ and the explicit expression of $(\mathcal{E}, \mathcal{F})$.

There are quite a few works [1,23-25] dealing with generalizations of Itô's one [15]. See Remark 2.2 and Remark 4.1 in these regards. But construction and uniqueness of a symmetric extension $X$ of a symmetric $X^{0}$ as are formulated in the present paper have never been considered. 


\section{Strongly local Dirichlet form with a point of positive capacity}

\subsection{Description of the form and resolvent by absorbed process}

Let $S$ be a locally compact separable metric space and $a$ be a non-isolated point of $S$. We denote the complementary set $S \backslash\{a\}$ by $S_{0}$. Let $m$ be a positive Radon measure on $S$ with $\operatorname{Supp}[m]=S$ and with $m(\{a\})=0$. The inner product in each of the spaces $L^{2}(S ; m), L^{2}\left(S_{0}, m\right)$ will be designated by $(\cdot, \cdot)$.

A Dirichlet form $(\mathcal{E}, \mathcal{F})$ on $L^{2}(S ; m)$ is called regular if $\mathcal{F} \cap C_{0}(S)$ is $\mathcal{E}_{1}$-dense in $\mathcal{F}$ and uniformly dense in $C_{0}(S)$, where $C_{0}(S)$ denotes the space of continuous functions on $S$ with compact support. It is called strongly local if $\mathcal{E}(u, v)$ vanishes whenever $u, v \in \mathcal{F}, \operatorname{Supp}[u], \operatorname{Supp}[v]$ are compact and $v$ is constant on a neighbourhood of Supp $[u]$, where $\operatorname{Supp}[u]$ denotes the topological support of the measure $u \cdot m$. For the sake of a use in $\S 3.2$, we make here a remark:

Remark 2.1. If a Dirichlet form $(\mathcal{E}, \mathcal{F})$ on $L^{2}(S ; m)$ is regular and strongly local, then the strong locality stated above holds without assuming that $\operatorname{Supp}[v]$ is compact. Indeed, assuming the boundedness of $v$, take a function $w \in \mathcal{F} \cap C_{0}(S)$ with $w=1$ on a neighbourhood of $K=\operatorname{Supp}[u]$ and put $v_{1}=v \cdot w, v_{0}=v-v_{1}$. Then $\mathcal{E}\left(u, v_{1}\right)=0$. Since $v_{0}$ belongs to the part $\mathcal{F}_{G}$ of $(\mathcal{E}, \mathcal{F})$ on the open set $G=S \backslash K$ and $\left(\mathcal{E}, \mathcal{F}_{G}\right)$ is a regular Dirichlet form on $L^{2}(G ; m)$ (cf. [9, Theorem 4.4.3]), we can find $v_{n} \in \mathcal{F} \cap C_{0}(G)$ which are $\mathcal{E}_{1}$-convergent to $v_{0}$. Hence $\mathcal{E}\left(u, v_{0}\right)=$ $\lim _{n \rightarrow \infty} \mathcal{E}\left(u, v_{n}\right)=0$ and $\mathcal{E}(u, v)=0$.

We consider a strongly local regular Dirichlet form $(\mathcal{E}, \mathcal{F})$ on $L^{2}(S ; m)$ and an associated $m$-symmetric Hunt process $X=\left(X_{t}, P_{x}\right)$ on $S$. In view of [9, Theorem 4.5.3], $X$ can then be taken to be a diffusion on $S_{\Delta}$ in the sense that all sample paths are continuous functions from $[0, \infty)$ to $S_{\Delta}$, where $S_{\Delta}$ is the one-point compactification of $S$ when $S$ is non-compact and $\Delta$ is an extra point isolated from $S$ when $S$ is compact. In either case $\Delta$ will be the cemetery of the sample paths. Furthermore, $X$ can be taken to be of no killing inside $S$ in the sense that

$$
P_{x}\left(X_{\zeta-}=\Delta, \zeta<\infty\right)=P_{x}(\zeta<\infty), \quad x \in S,
$$

where $\zeta(\omega)$ denotes the life time, namely, the hitting time of the cemetery $\Delta$ of the sample path $\omega$. In particular, when $S$ is compact, $P_{x}(\zeta=\infty)=1$ for all $x \in S$.

We make the assumption that

\section{B.1 $\operatorname{Cap}(\{a\})>0$.}

Here $\operatorname{Cap}(A)$ for $A \subset S$ is its 1 -capacity relative to $(\mathcal{E}, \mathcal{F})$. In what follows, the quasi-continuity of functions on $S$ will be understood with respect to this capacity. Each function $u \in \mathcal{F}$ admits its quasi-continuous version denoted by $\tilde{u}$. 'q.e.' will means 'except for a set of zero capacity'.

The hitting probability and the $\alpha$-order hitting probability of $\{a\}$ are denoted by $\varphi$ and $u_{\alpha}$ respectively:

$$
\varphi(x)=P_{x}(\sigma<\infty), \quad u_{\alpha}(x)=E_{x}\left(\mathrm{e}^{-\alpha \sigma}\right), \quad x \in S,
$$

where $\sigma$ is the hitting time of $a$ by the process $X$ defined by

$$
\sigma=\inf \left\{t>0: X_{t}=a\right\} .
$$

The assumption B.1 implies that $u_{\alpha}$ is a non-trivial element of $\mathcal{F}$ and it is the $\alpha$-potential $U_{\alpha} v_{\alpha}$ of a positive measure $v_{\alpha}$ concentrated on $\{a\}$ (cf. $\left.[9, \S 2.2]\right)$ :

$$
\mathcal{E}_{\alpha}\left(u_{\alpha}, v\right)=\tilde{v}(a) v_{\alpha}(\{a\}), \quad v \in \mathcal{F} .
$$

Put

$$
\mathcal{F}_{0}=\{u \in \mathcal{F}: \tilde{u}(a)=0\} .
$$


Then $\left(\mathcal{E}, \mathcal{F}_{0}\right)$ is a regular strongly local Dirichlet form on $L^{2}\left(S_{0} ; m\right)$, which is associated with the part $X^{0}=$ $\left(X_{t}^{0}, P_{x}^{0}\right)$ of $X$ on the set $S_{0}$, namely, the diffusion process $X^{0}$ obtained from $X$ by killing upon the hitting time $\sigma$ (cf. $[9, \S 4.4]) . X^{0}$ is of no killing inside $S_{0}$ and, if we denote the life time of $X^{0}$ by $\zeta^{0}$, then $\varphi, u_{\alpha}$ admit the expressions

$$
\varphi(x)=P_{x}^{0}\left(\zeta^{0}<\infty, X_{\zeta^{0}-}^{0}=a\right), \quad u_{\alpha}(x)=E_{x}^{0}\left(\mathrm{e}^{-\alpha \zeta^{0}} ; X_{\zeta^{0}-}=a\right), \quad x \in S_{0},
$$

in terms of the absorbed process $X^{0}$. We further consider the functions

$$
\psi^{(1)}(x)=P_{x}^{0}\left(\zeta^{0}<\infty, X_{\zeta^{0}-}=\Delta\right), \quad \psi^{(2)}(x)=P_{x}^{0}\left(\zeta^{0}=\infty\right), \quad x \in S_{0},
$$

and put $\psi=\psi^{(1)}+\psi^{(2)}$ so that $\psi=1-\varphi$.

Denote by $p_{t}$ and $G_{\alpha}$ the transition function and the resolvent of $X$ respectively. The same notions for the absorbed process $X^{0}$ will be denoted by $p_{t}^{0}$ and $G_{\alpha}^{0}$. The functions $\varphi, \psi^{(1)}, \psi^{(2)}$ on $S_{0}$ are $X^{0}$-excessive. In particular, $\psi^{(2)}$ is $X^{0}$-invariant in the sense that $\psi^{(2)}=p_{t}^{0} \psi^{(2)}, t>0$. Because of the $m$-symmetry of $X^{0}$, the measure

$$
m_{0}=\varphi \cdot m
$$

is an $X^{0}$-excessive measure with $m_{0} p_{t}^{0}=p_{t}^{0} \varphi \cdot m$.

Our first aim in this section is to show under the present setting that the form $\mathcal{E}$ as well as the resolvent $G_{\alpha}$ are uniquely and explicitly determined by quantities depending only on the absorbed process $X^{0}$.

We prepare a lemma.

Lemma 2.1. For an $X^{0}$-excessive function $v$ on $S_{0}$,

$$
L\left(m_{0}, v\right)=\lim _{t \downarrow 0} \frac{1}{t}\left\langle m_{0}-m_{0} p_{t}^{0}, v\right\rangle=\lim _{t \downarrow 0} \frac{1}{t}\left(\varphi-p_{t}^{0} \varphi, v\right)(\leqslant \infty) .
$$

is well defined as an increasing limit and it holds that

$$
L\left(m_{0}, v\right)=\lim _{\alpha \rightarrow \infty} \alpha\left(u_{\alpha}, v\right) .
$$

If $v$ is $p_{t}^{0}$-invariant, then for each $t>0$ and $\alpha>0$,

$$
L\left(m_{0}, v\right)=\frac{1}{t}\left(\varphi-p_{t}^{0} \varphi, v\right)=\alpha\left(u_{\alpha}, v\right) .
$$

Proof. If we set $e(t)=\left(\varphi-p_{t}^{0} \varphi, v\right)$, then

$$
e(t+s)=e(t)+\left(p_{t}^{0} \varphi-p_{t+s}^{0} \varphi, v\right)=e(t)+\left(\varphi-p_{s}^{0} \varphi, p_{t}^{0} v\right) \leqslant e(t)+e(s),
$$

and hence $e(t) / t$ is increasing as $t$ decreases and constant if $v$ is $p_{t}^{0}$-invariant. We also see that

$$
\alpha\left(u_{\alpha}, v\right)=\alpha\left(\varphi-\alpha G_{\alpha}^{0} \varphi, v\right)=\int_{0}^{\infty} \mathrm{e}^{-t}(t / \alpha)^{-1}\left(\varphi-p_{t / \alpha}^{0} \varphi, v\right) t \mathrm{~d} t
$$

increases to $L(v)$ as $\alpha \uparrow \infty$.

We note that $L\left(m_{0}, v\right)$ is nothing but the energy functional of the $X^{0}$-excessive measure $m_{0}$ and the $X^{0}$-excessive function $v$ in the sense of P.A. Meyer [21] when $X^{0}$ is transient (cf. [4, §39], [11, p. 16]). In [4, §39], it is called the mass of $v$ relative to $m_{0}$.

Let $\mathcal{F}_{e}$ (resp. $\left.\mathcal{F}_{0, e}\right)$ be the extended Dirichlet space of $(\mathcal{F}, \mathcal{E})\left(\operatorname{resp} .\left(\mathcal{F}_{0}, \mathcal{E}\right)\right.$ ). Each element $u \in \mathcal{F}_{e}$ admits its quasi continuous version denoted by $\tilde{u}$ again. In view of $[9, \S 4.6]$, it holds then that 


$$
\begin{aligned}
& \mathcal{F}_{0, e}=\mathcal{F}_{e, 0}=\left\{u \in \mathcal{F}_{e}: \tilde{u}(a)=0\right\}, \\
& \varphi \in \mathcal{F}_{e}, \quad \mathcal{E}(\varphi, u)=0 \quad \forall u \in \mathcal{F}_{e, 0}, \\
& \mathcal{F}=\mathcal{F}_{e} \cap L^{2}(S, m), \quad \mathcal{F}_{0}=\mathcal{F}_{0, e} \cap L^{2}\left(S_{0}, m\right) .
\end{aligned}
$$

Furthermore any $w \in \mathcal{F}_{e}$ can be decomposed as

$$
w=u_{0}+c \varphi, \quad u_{0} \in \mathcal{F}_{e, 0}, \quad c \text { constant }
$$

and

$$
\mathcal{E}(w, w)=\mathcal{E}\left(u_{0}, u_{0}\right)+c^{2} \mathcal{E}(\varphi, \varphi)
$$

\section{Theorem 2.1.}

(i) It holds that

$$
\mathcal{E}(\varphi, \varphi)=L\left(m_{0}, \psi\right) \quad\left(=L\left(m_{0}, \psi^{(1)}\right)+L\left(m_{0}, \psi^{(2)}\right)\right) .
$$

(ii) $u_{\alpha}$ is a non-trivial element of $\mathcal{F} \cap L^{1}\left(S_{0} ; m\right)$.

(iii) For any $f \in L^{2}(S, m)$ and $x \in S$,

$$
G_{\alpha} f(x)=G_{\alpha}^{0} f(x)+\frac{\left(u_{\alpha}, f\right)}{\alpha\left(u_{\alpha}, \varphi\right)+L\left(m_{0}, \psi\right)} u_{\alpha}(x), \quad G_{\alpha} f(a)=\frac{\left(u_{\alpha}, f\right)}{\alpha\left(u_{\alpha}, \varphi\right)+L\left(m_{0}, \psi\right)} .
$$

(iv) Let $\delta_{a}$ be a unit mass concentrated at $\{a\}$. Then it is of finite energy integral and its $\alpha$-potential $U_{\alpha} \delta_{a}$ is related to $u_{\alpha}$ by

$$
\widetilde{U_{\alpha} \delta_{a}}=\frac{1}{\alpha\left(u_{\alpha}, \varphi\right)+L\left(m_{0}, \psi\right)} u_{\alpha}
$$

(v) The point a is regular for itself and also an instantaneous state with respect to $X$ :

$$
P_{a}\left(\sigma=0, \tau_{a}=0\right)=1, \quad \tau_{a}=\inf \left\{t>0: X_{t} \in S_{0}\right\} .
$$

Proof. We first give a proof of (ii). According to a general theorem [9, Chapter 4], the formula obtained by the strong Markov property

$$
G_{\alpha} f(x)=G_{\alpha}^{0} f(x)+u_{\alpha}(x) G_{\alpha} f(a), \quad x \in S, f \in L^{2}(S, m),
$$

represents the orthogonal decomposition of $G_{\alpha} f \in \mathcal{F}$ into the space $\mathcal{F}_{0}$ and its orthogonal complement $\mathcal{H}_{\alpha}=$ $\left\{c \cdot u_{\alpha}: c\right.$ constant $\}$ in the Hilbert space $\left(\mathcal{F} \cdot \mathcal{E}_{\alpha}\right)$. We see that $G_{\alpha} f(a)>0$ for some $f \in C_{0}^{+}(S)$, because otherwise $\mathcal{F}=\mathcal{F}^{0}$ from (2.18) contradicting to $u_{\alpha} \in \mathcal{F}$. By (2.18),

$$
\left(u_{\alpha}, 1\right) G_{\alpha} f(a) \leqslant\left(G_{\alpha} f, 1\right)=\left(f, G_{\alpha} 1\right) \leqslant \frac{1}{\alpha}(f, 1)<\infty .
$$

Next we prove (i) and (iii). For $f \in C_{0}(S)$, the function $w=G_{\alpha} f$ has two expressions:

$$
w=G_{\alpha}^{0} f+c u_{\alpha}=u_{0}+c \varphi, \quad c=G_{\alpha} f(a), u_{0} \in \mathcal{F}_{e, 0} .
$$

By [9, Corollary 1.6.3, Theorem 2.1.7], we can find a sequence $\left\{g_{n}\right\}$ of uniformly bounded functions in $\mathcal{F}$ such that

$$
\lim _{n \rightarrow \infty} g_{n}=\varphi \quad m \text {-a.e., } \quad \lim _{n \rightarrow \infty} \mathcal{E}\left(g_{n}-\varphi, g_{n}-\varphi\right)=0 .
$$

Letting $n \rightarrow \infty$ in the equation

$$
\mathcal{E}\left(w, g_{n}\right)+\alpha\left(w, g_{n}\right)=\left(f, g_{n}\right),
$$


we get

$$
c \mathcal{E}(\varphi, \varphi)+c \alpha\left(u_{\alpha}, \varphi\right)=(f, \varphi)-\left(\alpha G_{\alpha}^{0} f, \varphi\right) .
$$

Since the right-hand side equals

$$
\left(f, \varphi-\alpha G_{\alpha}^{0} \varphi\right)=\left(f, u_{\alpha}\right),
$$

we arrive at

$$
G_{\alpha} f(a)=\frac{\left(u_{\alpha}, f\right)}{\alpha\left(u_{\alpha}, \varphi\right)+\mathcal{E}(\varphi, \varphi)}, \quad f \in C_{0}(S) .
$$

(2.19) holds for any bounded Borel $f$. In particular, we have for any $\alpha>0$,

$$
G_{\alpha} 1(a)=\frac{\left(u_{\alpha}, 1\right)}{\alpha\left(u_{\alpha}, \varphi\right)+\mathcal{E}(\varphi, \varphi)} \leqslant \frac{1}{\alpha},
$$

and hence

$$
\mathcal{E}(\varphi, \varphi) \geqslant \alpha\left(u_{\alpha}, \psi\right) .
$$

By letting $\alpha \rightarrow \infty$, we get from Lemma 2.1

$$
\mathcal{E}(\varphi, \varphi) \geqslant L\left(m_{0}, \psi\right)
$$

In order to prove (2.14), notice that the assumption of the strong locality of $\mathcal{E}$ implies that the killing measure $k$ in the Beurling-Deny representation of $\mathcal{E}$ vanishes (cf. [9, Theorem 4.5.3]). On account of [9, Lemma 4.5.2],

$$
\int_{S} f^{2} \mathrm{~d} k=\lim _{\alpha \rightarrow \infty} \alpha \int_{S} f(x)^{2}\left(1-\alpha G_{\alpha} 1(x)\right) m(\mathrm{~d} x), \quad f \in \mathcal{F} \cap C_{0}(S) .
$$

From (2.18) and (2.19), we have

$$
\begin{aligned}
1-\alpha G_{\alpha} 1(x) & =1-\alpha G_{\alpha}^{0} 1(x)-\frac{\alpha\left(u_{\alpha}, 1\right)}{\alpha\left(u_{\alpha}, \varphi\right)+\mathcal{E}(\varphi, \varphi)} u_{\alpha}(x) \geqslant u_{\alpha}(x)-\frac{\alpha\left(u_{\alpha}, 1\right)}{\alpha\left(u_{\alpha}, \varphi\right)+\mathcal{E}(\varphi, \varphi)} u_{\alpha}(x) \\
& =\frac{\mathcal{E}(\varphi, \varphi)-\alpha\left(u_{\alpha}, \psi\right)}{\alpha\left(u_{\alpha}, \varphi\right)+\mathcal{E}(\varphi, \varphi)} u_{\alpha}(x) .
\end{aligned}
$$

Take $f \in \mathcal{F} \cap C_{0}(S)$ such that $f(a) \neq 0$. We have from (2.19) and the above inequality

$$
\alpha \int_{S} f^{2}\left(1-\alpha G_{\alpha} 1\right) \mathrm{d} m \geqslant\left(\mathcal{E}(\varphi, \varphi)-\alpha\left(u_{\alpha}, \psi\right)\right)\left(\alpha G_{\alpha} f^{2}\right)(a) .
$$

By letting $\alpha \rightarrow \infty$, we get

$$
0 \geqslant\left(\mathcal{E}(\varphi, \varphi)-L\left(m_{0}, \psi\right)\right) f(a)^{2},
$$

proving the desired identity (2.14).

Proof of (iv). By (2.3),

$$
\left(u_{\alpha}, f\right)=\mathcal{E}_{\alpha}\left(u_{\alpha}, G_{\alpha} f\right)=G_{\alpha} f(a) v_{\alpha}(\{a\}),
$$

which combined with (2.15) gives

$$
v_{\alpha}=\left(\alpha\left(u_{\alpha}, \varphi\right)+L\left(m_{0}, \psi\right)\right) \delta_{a} .
$$

Proof of (v). The regularity $P_{a}(\sigma=0)=1$ of the point $a$ for itself follows from A.1 and a general fact that, for any Borel set $B$, the set of irregular points $x \in B$ for $B$ is of zero capacity [9, Chapter 4]. If $P_{a}\left(0<\tau_{a}<\infty\right)>0$, then $P_{a}\left(X_{\tau_{a}} \in S_{0} \cup \Delta\right)=1$ contradicting the sample continuity and absence of the killing inside $S$ for $X$. If $a$ were a trap with respect to $X$, then $G_{\alpha} f(a)=f(a) / \alpha$ for any $f \in L^{2}(S ; m)$ contradicting (2.15). Accordingly, $a$ is an instantaneous state. 
Remark 2.2. (i) The present assumptions can be relaxed as follows:

(a) The measure $m$ on $S$ is replaced by $\bar{m}=m+\gamma \delta_{a}$ for a non-negative constant $\gamma$.

(b) $(\mathcal{E}, \mathcal{F})$ is assumed to be a (not necessarily strongly) local regular Dirichlet form on $L^{2}(S ; \bar{m})$, while its part $\left(\mathcal{E}, \mathcal{F}_{0}\right)$ on $S_{0}$ is assumed to be a strongly local Dirichlet form on $L^{2}\left(S_{0} ; m\right)$.

Then, in view of the above proof of Theorem 2.1, we readily see that (2.14) and (2.15) remain true under the following modifications:

$$
\begin{aligned}
& \mathcal{E}(\varphi, \varphi)=L\left(m_{0}, \psi\right)+\delta, \\
& G_{\alpha} f(x)=G_{\alpha}^{0} f(x)+\frac{\left(u_{\alpha}, f\right)+\gamma f(a)}{\alpha\left(u_{\alpha}, \varphi\right)+L\left(m_{0}, \psi\right)+\delta+\alpha \gamma} u_{\alpha}(x),
\end{aligned}
$$

for a non-negative constant $\delta$.

Example 6.1 will indicate stochastic interpretations of the parameters $\gamma$ and $\delta$.

(ii) The parameters $\gamma, \delta$ have appeared in Rogers' description [23] of the most general extension of a general resolvent $G_{\alpha}^{0}$ under a setting corresponding to $\psi^{(1)}=0$. Another parameter appearing in [23] is a family of measures $n_{\alpha}, \alpha>0$, on $S_{0}$, which is reduced to $u_{\alpha} \cdot m$ under the present symmetry assumption.

(iii) In the setting (i) in the above, $G_{\alpha}$ is conservative if and only if $\psi^{(1)}=0$ and $\delta=0$, and in this case the above expression is reduced to

$$
G_{\alpha} f(x)=G_{\alpha}^{0} f(x)+\frac{\left(1-\alpha G_{\alpha}^{0} 1, f\right)+\gamma f(a)}{\alpha\left(1-\alpha G_{\alpha}^{0} 1,1\right)+\alpha \gamma}\left(1-\alpha G_{\alpha}^{0} 1(x)\right) .
$$

Such a formula was found by Y. Le Jan [18] (see also [4, §78]) in a general setting to produce conservative resolvents out of a (not necessarily symmetric) sub-Markovian resolvent and its dual preserving the duality.

\subsection{Description of the inverse local time}

In $\S 4$, we shall construct a diffusion on $S$ with resolvent (2.15) by means of Poisson point processes of excursions, namely, by piecing together the excursions. In this subsection, let us study more about the roles of the measure $m_{0}$ and the energy functional $L\left(m_{0}, \psi\right)$ played in the present diffusion $X$ on $S$.

Let $L(t)$ be the positive continuous additive functional (admitting exceptional set) associated with the smooth measure $\delta_{a}$ (cf. [9, §5.1]):

$$
\widetilde{U_{\alpha} \delta_{a}}(x)=E_{x}\left(\int_{0}^{\infty} \mathrm{e}^{-\alpha t} \mathrm{~d} L(t)\right) \text { for q.e. } x \in S .
$$

In particular, (2.20) holds for $x=a . L(t)$ is a local time at $\{a\}$ in the sense that it increases only when $X_{t}=a$ :

$$
L(t)=\int_{0}^{t} I_{a}\left(X_{s}\right) \mathrm{d} L(s)
$$

We consider the right continuous inverse $S(t)=\inf \{s: L(s)>t\}$ of $L(t)$.

It is well known that the increasing process $\left(S(t), P_{a}\right)$ is a subordinator killed upon an exponential holding time (cf. [2]). Theorem 2.1 enables us to identify the Lévy measure of the subordinator and the killing rate. Indeed, according to [2, v (3.17)], (2.20) implies the identity

$$
E_{a}\left(\mathrm{e}^{-\alpha S(t)}\right)=\exp \left(-t / \widetilde{U_{\alpha} \delta_{a}}(a)\right),
$$

which combined with (2.16) leads us to

$$
E_{a}\left(\mathrm{e}^{-\alpha S(t)}\right)=\mathrm{e}^{-t L\left(m_{0}, \psi\right)} \exp \left[-t \alpha\left(u_{\alpha}, \varphi\right)\right] .
$$


We need a lemma which will play a basic role in $\S 4$ again. A family $\left\{v_{t}\right\}_{t>0}$ of $\sigma$-finite measures on $S_{0}$ is called an $X^{0}$-entrance law if $v_{t} p_{s}^{0}=v_{s+t}, s, t>0$. Then $v_{t}(f), f \in \mathcal{B}^{+}\left(S_{0}\right)$, is measurable in $t$ and we may let

$$
\hat{v}_{\alpha}(f)=\int_{0}^{\infty} \mathrm{e}^{-\alpha t} v_{t}(f) \mathrm{d} t, \quad \alpha>0, f \in \mathcal{B}^{+}\left(S_{0}\right) .
$$

\section{Lemma 2.2.}

(i) There exists a unique $X^{0}$-entrance law $\left\{\mu_{t}\right\}$ such that

$$
m_{0}=\int_{0}^{\infty} \mu_{t} \mathrm{~d} t
$$

(ii) $\hat{\mu}_{\alpha}(f)=\left(u_{\alpha}, f\right), \alpha>0, f \in \mathcal{B}^{+}\left(S_{0}\right)$. Consequently,

$$
\int_{0}^{t} \mu_{s}(f) \mathrm{d} s=\int_{S_{0}} P_{x}^{0}\left(\zeta^{0} \leqslant t, X_{\zeta^{0}-}=a\right) f(x) m(\mathrm{~d} x), \quad t>0, f \in \mathcal{B}\left(S_{0}\right) .
$$

(iii) $\mu_{t}\left(S_{0}\right)<\infty, t>0$.

(iv) For any bounded $X^{0}$-excessive function $v$ on $S_{0}, \mu_{t}(v)$ is right continuous in $t>0$.

(v) For any $X^{0}$-excessive function $v$ on $S_{0}$, the energy functional $L\left(m_{0}, v\right)$ introduced in Lemma 2.1 admits an expression

$$
L\left(m_{0}, v\right)=\lim _{t \downarrow 0} \mu_{t}(v) .
$$

When $v$ is $p_{t}^{0}$-invariant, it holds for any $t>0$ that

$$
L\left(m_{0}, v\right)=\mu_{t}(v) .
$$

(vi) $L\left(m_{0}, \varphi\right)=\infty$.

Proof. (i) Since

$$
p_{t}^{0} \varphi(x)=P_{x}^{0}\left(t<\zeta^{0}<\infty, X_{\zeta-}^{0}=a\right) \downarrow 0, \quad t \rightarrow \infty,
$$

$\lim _{t \downarrow 0} m_{0} p_{t}^{0}(f)=\left(p_{t}^{0} \varphi, f\right)=0$ for $f \in L^{1}\left(S_{0}, m\right)$, namely, $m_{0}$ is purely excessive. Hence the desired assertion follows from a well known representation theorem provided that $X^{0}$ is transient [11, Theorem 5.25]. But the present situation can be reduced to this case by observing that

$$
S_{1}=\left\{x \in S_{0}: \varphi(x)>0\right\}
$$

is a non-trivial $X^{0}$-invariant set q.e. and the restriction of $X^{0}$ to $S_{1}$ is transient (cf. $[9, \S 4.6]$ ).

(ii) For $f \in C_{0}^{+}\left(S_{0}\right)$, we have

$$
\int_{t}^{\infty} \mu_{t}(f) \mathrm{d} t=\int_{0}^{\infty} \mu_{t+s}(f) \mathrm{d} t=\int_{0}^{\infty} \mu_{s}\left(p_{t}^{0} f\right) \mathrm{d} s=\left(\varphi, p_{t}^{0} f\right),
$$

and

$$
\mu_{t}(f)=-\frac{\mathrm{d}}{\mathrm{d} t}\left(\varphi, p_{t}^{0} f\right), \quad \text { a.e. } t
$$


Hence

$$
\begin{aligned}
\hat{\mu}_{\alpha}(f) & =-\int_{0}^{\infty} \mathrm{e}^{-\alpha t} \frac{d}{\mathrm{~d} t}\left(\varphi, p_{t}^{0} f\right) \mathrm{d} t=\left[-\mathrm{e}^{-\alpha t}\left(\varphi, p_{t}^{0} f\right)\right]_{0}^{\infty}-\alpha \int_{0}^{\infty} \mathrm{e}^{-\alpha t}\left(\varphi, p_{t}^{0} f\right) \mathrm{d} t \\
& =(\varphi, f)-\alpha\left(\varphi, G_{\alpha}^{0} f\right)=\left(\varphi-\alpha G_{\alpha}^{0} \varphi, f\right)=\left(u_{\alpha}, f\right) .
\end{aligned}
$$

(iii) By (ii) and Theorem 2.1 (ii), $\hat{\mu}_{\alpha}(1)=\left(u_{\alpha}, 1\right)<\infty$, from which the desired finiteness follows.

(iv) On account of (iii), we have $\mu_{t+s}(v)=\mu_{t}\left(p_{s}^{0} v\right) \rightarrow \mu_{t}(v), s \downarrow 0$.

(v) Since $\left\langle\mu_{t}, v\right\rangle$ is increasing as $t \downarrow 0$ (independent of $t$ when $v$ is $p_{t}^{0}$-invariant), the assertions follow from

$$
\left\langle m_{0}-m_{0} p_{t}^{0}, v\right\rangle=\int_{0}^{t}\left\langle\mu_{s}, v\right\rangle \mathrm{d} s .
$$

(vi) Since $S(t)$ is the right continuous inverse of an increasing continuous process $L(t), P_{a}(S(t)>0)=1$ and consequently we have

$$
L\left(m_{0}, \varphi\right)=\lim _{\alpha \rightarrow \infty} \alpha\left(u_{\alpha}, \varphi\right)=\infty
$$

by letting $\alpha \rightarrow \infty$ in (2.21).

We see by the above lemma that $\mu_{t}(\varphi)$ is decreasing and right continuous in $t>0$ and so we can define a measure $\Theta$ on $(0, \infty)$ by

$$
\Theta((s, t])=\mu_{s}(\varphi)-\mu_{t}(\varphi), \quad 0<s<t .
$$

It then holds that

$$
\Theta((s, t])=\mu_{s}\left(\varphi-p_{t-s}^{0} \varphi\right)=\left\langle\mu_{s}, P .(\sigma \leqslant t-s)\right\rangle,
$$

and we get by letting $t \rightarrow \infty$,

$$
\Theta((s, \infty))=\mu_{s}(\varphi)
$$

We note that

$$
\Theta([\delta, \infty))<\infty
$$

for each $\delta>0$ by virtue of Lemma 2.2 (iii).

\section{Lemma 2.3. It holds that}

$$
\alpha\left(u_{\alpha}, \varphi\right)=\int_{0}^{\infty}\left(1-\mathrm{e}^{-\alpha u}\right) \Theta(\mathrm{d} u)
$$

Proof. We have from Lemma 2.2 (ii) and (2.25)

$$
\alpha\left(u_{\alpha}, \varphi\right)=\alpha \hat{\mu}_{\alpha}(\varphi)=\alpha \int_{0}^{\infty} \mathrm{e}^{-\alpha t} \Theta((t, \infty)) \mathrm{d} t=\int_{0}^{\infty} \int_{0}^{s} \alpha \mathrm{e}^{-\alpha t} \mathrm{~d} t \Theta(\mathrm{d} s)=\int_{0}^{\infty}\left(1-\mathrm{e}^{-\alpha s}\right) \Theta(\mathrm{d} s) .
$$

On account of the formula (2.21), Lemma 2.3 and by noting that $\lim _{\alpha \downarrow} \alpha\left(u_{\alpha}, \varphi\right)=0$, we can get the next theorem from [2, Theorem 3.21]. 
Theorem 2.2. Define a measure $\Theta$ on $(0, \infty)$ by $(2.24)$. On a certain probability space $(\Omega, \mathcal{B}, P)$, construct a subordinator $\left\{Y_{t}\right\}_{t} \geqslant 0$ with Lévy measure $\Theta$ and zero drift and a random variable $Z$, independent of $\left\{Y_{t}\right\}$, with

$$
P(Z \geqslant t)=\mathrm{e}^{-L\left(m_{0}, \psi\right) t}, \quad t \geqslant 0 .
$$

If we let

$$
S^{*}(t)= \begin{cases}Y(t), & t<Z \\ \infty, & t \geqslant Z\end{cases}
$$

then the process $\left(\left\{S^{*}(t)\right\}_{t \geqslant 0}, P\right)$ is equivalent in law to $\left(\{S(t)\}_{t \geqslant 0}, P_{a}\right)$.

\section{Strongly local Dirichlet form with a recurrent point}

Let $S$ and $m$ be as in $\S 2$. In this section, we consider a special case of the Dirichlet form of $\S 2$ for which the point $a$ is recurrent.

\subsection{Description of associated Poisson point process and entrance law}

Let $(\mathcal{E}, \mathcal{F})$ be a strongly local regular Dirichlet form on $L^{2}(S ; m)$ and $X=\left(X_{t}, P_{x}\right)$ be an associated diffusion on $S$. In place of the assumption B.1 of $\S 2$, let us assume that

B.2 $\varphi(x)>0, m$-a.e. $x \in S_{0}$;

B.3 $1 \in \mathcal{F}_{e}$ and $\mathcal{E}(1,1)=0$.

In the next subsection, we shall construct a typical example of a Dirichlet form $(\mathcal{E}, \mathcal{F})$ satisfying these conditions by a method of the one point compactification.

The assumption B.2 implies that $u_{1}>0, m$-a.e. and $\operatorname{Cap}(\{a\})=\mathcal{E}_{1}\left(u_{1}, u_{1}\right) \geqslant\left(u_{1}, u_{1}\right)>0$, namely, the assumption B.1 of $\S 1$ (cf. [9, Lemma 4.2.1]). Further, the Dirichlet form $(\mathcal{E}, \mathcal{F})$ becomes irreducible because, from $(2.15)$, we have for any Borel sets $B_{1}, B_{2} \subset S$ of positive $m$-measures

$$
\left(I_{E}, G_{\alpha} I_{F}\right) \geqslant\left(u_{\alpha}, I_{E}\right)\left(u_{\alpha}, I_{F}\right) / \alpha\left(u_{\alpha}, \varphi\right)>0 .
$$

Since $(\mathcal{E}, \mathcal{F})$ is recurrent by B.3, we have actually the property

$$
\varphi(x)=1, \quad \text { q.e. } x \in S,
$$

stronger than the assumption B.2 in view of [9, Theorem 4.6.6].

Thus the point $a$ is not only regular for itself, instantaneous, but also recurrent. (2.15) is now reduced to

$$
G_{\alpha} f(x)=G_{\alpha}^{0} f(x)+\frac{\left(u_{\alpha}, f\right)}{\alpha\left(u_{\alpha}, 1\right)} u_{\alpha}(x), \quad x \in S, \quad G_{\alpha} f(a)=\frac{\left(u_{\alpha}, f\right)}{\alpha\left(u_{\alpha}, 1\right)} .
$$

The positive continuous additive functional $L(t)$ of $X$ associated with the unit mass $\delta_{a}$ has the property that $L(\infty)=\infty$ and its right continuous inverse $S(t)$ is a subordinator satisfying

$$
E_{a}\left(\int_{0}^{\infty} \mathrm{e}^{-\alpha S(s)} \mathrm{d} s\right)=\frac{1}{\alpha\left(u_{\alpha}, 1\right)}
$$

on account of (2.16) and (2.20).

Therefore we can follow directly the argument of $[15, \S 6$, case $2(b)]$ to conclude that 


$$
\begin{aligned}
& D_{\mathbf{p}}=\{s: S(s)-S(s-)>0\} \\
& \mathbf{p}_{s}(t)=X_{S(s-)+t}, s \in D_{\mathbf{p}}, 0 \leqslant t<S(s)-S(s-),
\end{aligned}
$$

defines, under the law $P_{a}$, a $W_{a}$-valued Poisson point process $\mathbf{p}$, where $W_{a}$ is the space of continuous excursions in $S_{0}$ from $a$ to $a$ :

$$
W_{a}=\left\{w:[0, \zeta(\omega)) \rightarrow S_{0}, \text { continuous, } 0<\zeta(\omega)<\infty, w(0)=a, w(\zeta-)=a\right\} .
$$

Let $\mathbf{n}$ be the characteristic measure of the Poisson point process $\mathbf{p}$. Then $\mathbf{n}$ is a $\sigma$-finite measure on the space $W_{a}$ and $\{w(t), \mathbf{n}\}$ is Markovian with respect to the transition function $p_{t}^{0}$ of $X^{0}$. The entrance law $\left\{v_{t}\right\}$ associated with the characteristic measure $\mathbf{n}$ is defined by

$$
v_{t}(B)=\mathbf{n}\{w: \zeta(w)>t, w(t) \in B\}, \quad B \in \mathcal{B}(S), t>0 .
$$

Recall that we have already considered an $X^{0}$-entrance law $\left\{\mu_{t}\right\}$ specified by (2.22) which is now reduced to

$$
m=\int_{0}^{\infty} \mu_{t} \mathrm{~d} t
$$

The description (2.23) of $\left\{\mu_{t}\right\}$ now reads

$$
\int_{0}^{t} \mu_{s}(f) \mathrm{d} s=\int_{S_{0}} P_{x}^{0}\left(\zeta^{0} \leqslant t\right) f(x) m(\mathrm{~d} x), \quad t>0, f \in \mathcal{B}\left(S_{0}\right) .
$$

Theorem 3.1. $v_{t}=\mu_{t}, t>0$.

Proof. By virtue of Lemma 2.2, it suffices to show that

$$
\hat{v}_{\alpha}(f)=\left(u_{\alpha}, f\right), \quad f \in \mathcal{B}_{b}\left(S_{0}\right) .
$$

We make use of the next general formula

$$
E_{a}\left(\sum_{s \leqslant t} a\left(s, \mathbf{p}_{s}, \omega\right)\right)=E_{a}\left(\int_{W_{a} \times(0, t]} a(s, w, \omega) \mathbf{n}(\mathrm{d} w) \mathrm{d} s\right)
$$

holding for any non-negative predictable function $a(s, w, \omega)$ on $[0, \infty) \times W_{a} \times \Omega, \Omega$ being a filtered sample space on which the diffusion process $X$ is defined (cf. [14, p. 62]).

Since $m(\{a\})$ is assumed to be zero, $\int_{0}^{\infty} I_{a}\left(X_{t}\right) \mathrm{d} t=0, P_{a}$-almost surely. By (3.4) and (3.5), we have for $f \in B_{b}(S)$,

$$
\begin{aligned}
G_{\alpha} f(a) & =E_{a}\left(\int_{0}^{\infty} \mathrm{e}^{-\alpha t} f\left(X_{t}\right) \mathrm{d} t\right)=E_{a}\left(\sum_{s>0} \int_{S(s-)}^{S(s)} \mathrm{e}^{-\alpha t} f\left(X_{t}\right) \mathrm{d} t\right) \\
& =E_{a}\left(\sum_{s>0} \mathrm{e}^{-\alpha S(s-)} \int_{0}^{\zeta\left(\mathbf{p}_{s}\right)} \mathrm{e}^{-\alpha t} f\left(\mathbf{p}_{s}(t)\right) \mathrm{d} t\right) .
\end{aligned}
$$

We let

$$
\Gamma(w)=\int_{0}^{\zeta(w)} \mathrm{e}^{-\alpha t} f(w(t)) \mathrm{d} t
$$


$a(s, w, \omega)=\Gamma(w) \cdot \mathrm{e}^{-\alpha S(s-, \omega)}$ is then predictable and we get by (3.11)

$$
G_{\alpha} f(a)=E_{a}\left(\sum_{s>0} \mathrm{e}^{-\alpha S(s-)} \Gamma\left(\mathbf{p}_{s}\right)\right)=\int_{W_{a}} \Gamma(w) \mathbf{n}(\mathrm{d} w) \cdot \int_{0}^{\infty} E_{a}\left(\mathrm{e}^{-\alpha S(s)}\right) \mathrm{d} s .
$$

Since

$$
\int_{W_{a}} \Gamma(w) \mathbf{n}(\mathrm{d} w)=\hat{v}_{\alpha}(f)
$$

(3.2) and (3.3) lead us to the desired identity (3.10).

By Theorem 3.1 and [15, Theorem 6.3], the finite dimensional distribution of $\left\{W_{a}, \mathbf{n}\right\}$ can be described as follows:

$$
\int_{W_{a}} f_{1}\left(w\left(t_{1}\right)\right) f_{2}\left(w\left(t_{2}\right)\right) \cdots f_{n}\left(w\left(t_{n}\right)\right) \mathbf{n}(\mathrm{d} w)=\mu_{t_{1}} f_{1} p_{t_{2}-t_{1}}^{0} f_{2} \cdots p_{t_{n-1}-t_{n-2}}^{0} f_{n-1} p_{t_{n}-t_{n-1}}^{0} f_{n},
$$

for any $0<t_{1}<t_{2}<\cdots<t_{n-1}, t_{n}, f_{1}, f_{2}, \ldots, f_{n} \in B_{b}\left(S_{0}\right)$. Here we use the convention that $w \in W$ satisfies $w(t)=\Delta, \forall t \geqslant \zeta(w)$, and any function $f$ on $S_{0}$ is extended to $S_{0} \cup \Delta$ by setting $f(\Delta)=0$.

In $\S 4$, we shall start with an $m$-symmetric diffusion $X^{0}$ on $S_{0}$ and an expression like the above with $\mu_{t}$ being specified by (2.22). See $\S 4$ for the abbreviated notation appearing on the right-hand side of (3.12).

Actually Theorem 3.1 can be extended to a general case where condition B. 3 of the recurrence is not assumed as we shall see in Remark 4.2 at the end of $\S 4$.

We note that the excursion law around a regular point of a general Markov process can be also formulated in terms of Maisonneuve's exit system [5]. Some property of the integral in $t$ of the associated entrance law was investigated by R.K. Getoor [10].

\subsection{Construction of form by one-point compactification}

In this subsection, we start with a Dirichlet form with underlying space $S_{0}$ and extend it by the one-point compactification to a Dirichlet form with underlying space $S=S_{0} \cup a$ satisfying B.2 and B.3 (and consequently B.1).

Let $S_{0}$ be a locally compact separable metric space and $m$ be a bounded positive measure on $S_{0}$ with $\operatorname{Supp}[m]=S_{0}$. We consider a regular strongly local Dirichlet form $\left(\mathcal{E}, \mathcal{F}_{0}\right)$ on $L^{2}\left(S_{0} ; m\right)$ satisfying the Poincaré inequality:

$$
(u, u) \leqslant A \cdot \mathcal{E}(u, u), \quad u \in \mathcal{F}_{0}, \exists A>0 .
$$

Denote by $S=S_{0} \cup a$ the one-point compactification of $S_{0}$ and by $L^{2}(S ; m)\left(=L^{2}\left(S_{0} ; m\right)\right)$ the space of square integrable functions on $S$ with respect to $I_{S_{0}} \cdot m$. Let us introduce a space $(\mathcal{E}, \mathcal{F})$ by

$$
\begin{aligned}
& \mathcal{F}=\mathcal{F}_{0}+\text { constant functions on } S \\
& \mathcal{E}\left(w_{1}, w_{2}\right)=\mathcal{E}\left(f_{1}, f_{2}\right), \quad w_{1}=f_{1}+c_{1}, w_{2}=f_{2}+c_{2}, f_{i} \in \mathcal{F}_{0}, c_{i} \text { constant. }
\end{aligned}
$$

\section{Theorem 3.2.}

(i) $(\mathcal{E}, \mathcal{F})$ is a regular strongly local Dirichlet form on $L^{2}(S ; m)$ possessing as its core the space

$$
\mathcal{C}=\mathcal{C}_{0}+\text { constant functions on } S_{0},
$$

where $\mathcal{C}_{0}=\mathcal{F}_{0} \cap C_{0}\left(S_{0}\right)$.

(ii) $(\mathcal{E}, \mathcal{F})$ and the associated diffusion on $S$ satisfy B.2, B.3. 
Proof. (i) Suppose $f \in \mathcal{F}_{0}$ is a constant. By the regularity of $\left(\mathcal{E}, \mathcal{F}_{0}\right)$, there exist $f_{n} \in \mathcal{F}_{0} \cap C_{0}\left(S_{0}\right)$ which are $\mathcal{E}_{1}$-convergent to $f$. We have then $\mathcal{E}(f, f)=\lim _{n \rightarrow \infty} \mathcal{E}\left(f, f_{n}\right)=0$ on account of the strong locality of $\left(\mathcal{E}, \mathcal{F}_{0}\right)$ and Remark 2.1 stated in the beginning of $\S 2.1$. (3.13) then implies $f=0$ and the definition (3.14) and (3.15) makes sense.

If $w_{n}=f_{n}+c_{n} \in \mathcal{F}$ is an $\mathcal{E}_{1}$-Cauchy sequence, then $f_{n}$ is $\mathcal{E}_{1}$-convergent to some $f \in \mathcal{F}_{0}$ by (3.13) and hence $w_{n}$ is $\mathcal{E}_{1}$-convergent to $f+c$ for some constant $c$.

Clearly $\mathcal{C}$ is dense both in $\mathcal{F}$ and $C(S)$, namely, $(\mathcal{E}, \mathcal{F})$ is regular.

Suppose, for $w_{i}=f_{i}+c_{i} \in \mathcal{C}$, that $w_{1}$ is constant on a neighbourhood of $\operatorname{Supp}\left(w_{2}\right)$. When $c_{2}=0, \mathcal{E}\left(w_{1}, w_{2}\right)=$ 0 by the strong locality of $\left(\mathcal{E}, \mathcal{F}_{0}\right)$. When $c_{2} \neq 0$, the set $U=S \backslash \operatorname{Supp}\left(w_{2}\right)$ is either empty or a non-empty relatively compact open subset of $S_{0}$. In the former case, $f_{1}=0$ and $\mathcal{E}\left(w_{1}, w_{2}\right)=0$. In the latter case, $f_{2}=-c_{2}$ on $U$, while $\operatorname{Supp}\left(f_{1}\right) \subset U$ and $\mathcal{E}\left(w_{1}, w_{2}\right)=\mathcal{E}\left(f_{1}, f_{2}\right)=0$ again. Hence $(\mathcal{E}, \mathcal{F})$ is strongly local on account of [9, Theorem 3.1.2].

The Markov property

$$
w \in \mathcal{F} \Rightarrow v=(0 \vee w) \wedge 1 \in \mathcal{F}, \quad \mathcal{E}(v, v) \leqslant \mathcal{E}(w, w)
$$

is evident, because, for $w=f+c, w \in \mathcal{F}_{0}, c$ constant, we have $v=[(-c) \vee f] \wedge(1-c)+c$.

(ii) B.2 follows from the Poincaré inequality (3.13). Denote by $X$ and $X^{0}=\left(X_{t}^{0}, P_{x}^{0}, \zeta^{0}\right)$ the diffusions associated with $(\mathcal{E}, \mathcal{F})$ and $\left(\mathcal{E}, \mathcal{F}_{0}\right)$ respectively. Then $X^{0}$ is the part of $X$ on $S_{0}$ and hence

$$
\varphi(x)=P_{x}^{0}\left(\zeta^{0}<\infty\right), \quad x \in S_{0}
$$

Denote by $G^{0}$ the 0 -order resolvent operator of $X^{0}$. Since $m\left(S_{0}\right)<\infty$, (3.13) implies that $G^{0} 1 \in \mathcal{F}_{0}$ and

$$
E_{x}^{0}\left(\zeta^{0}\right)=G^{0} 1(x)<\infty \quad \text { q.e. }
$$

proving (3.1). It is obvious from (3.14), (3.15) that $1 \in \mathcal{F}$ and $\mathcal{E}(1,1)=0$.

$\left(\mathcal{E}, \mathcal{F}_{0}\right)$ is not necessarily irreducible on $S_{0}$, but $(\mathcal{E}, \mathcal{F})$ defined by $(3.14),(3.15)$ is irreducible recurrent on $S$ in view of the observation made in the preceding subsection. See Example 6.2.

\section{Construction of a symmetric extension via excursion valued Poisson point processes}

In this section, we start with an $m$-symmetric diffusion $X^{0}$ on $S_{0}$ and construct first an excursion law with which Poisson point processes of two different kinds of excursions around the point $a$ are associated. We then construct an $m$-symmetric diffusion $\widetilde{X}$ on $S=S_{0} \cup a$ by piecing together those excursions. The resolvent of the resulting diffusion $\widetilde{X}$ turns out to be identical with (2.15).

\subsection{An excursion law and its basic properties}

Let $S$ be a locally compact separable metric space and $a$ be a non-isolated point of $S$. We put $S_{0}=S \backslash\{a\}$. The one point compactification of $S$ is denoted by $S_{\Delta}$. When $S$ is compact already, $\Delta$ is added as an isolated point. Let $m$ be a positive Radon measure on $S_{0}$ with $\operatorname{Supp}[m]=S_{0} . m$ is extended to $S$ by setting $m(\{a\})=0$.

We assume that we are given an $m$-symmetric diffusion $X^{0}=\left(X_{t}^{0}, P_{x}^{0}\right)$ on $S_{0}$ with life time $\zeta^{0}$ satisfying the following:

A.1 $P_{x}^{0}\left(\zeta^{0}<\infty, X_{\zeta^{0}-}^{0} \in\{a\} \cup\{\Delta\}\right)=P_{x}^{0}\left(\zeta^{0}<\infty\right), \forall x \in S_{0}$.

We define the functions $\varphi, u_{\alpha}, \psi^{(1)}, \psi^{(2)}, \psi$ by (2.5) and (2.6), namely, for $x \in S_{0}$, 


$$
\begin{aligned}
& \varphi(x)=P_{x}^{0}\left(\zeta^{0}<\infty, X_{\zeta^{0}-}^{0}=a\right), \quad u_{\alpha}(x)=E_{x}^{0}\left(\mathrm{e}^{-\alpha \zeta^{0}} ; X_{\zeta^{0}-}=a\right), \\
& \psi=1-\varphi=\psi^{(1)}+\psi^{(2)}, \quad \psi^{(1)}(x)=P_{x}^{0}\left(\zeta^{0}<\infty, X_{\zeta^{0}-}=\Delta\right), \quad \psi^{(2)}(x)=P_{x}^{0}\left(\zeta^{0}=\infty\right) .
\end{aligned}
$$

Let us assume that

A.2 $\varphi(x)>0, \forall x \in S_{0}$,

and

A.3 $u_{\alpha} \in L^{1}\left(S_{0} ; m\right), \forall \alpha>0$.

Denote by $p_{t}^{0}, G_{\alpha}^{0}$ the transition function and the resolvent of $X^{0}$ respectively. Our last assumption concerns the regularity:

A.4 $u_{\alpha} \in C_{b}\left(S_{0}\right), G_{\alpha}^{0}\left(C_{b}\left(S_{0}\right)\right) \subset C_{b}\left(S_{0}\right), \alpha>0$,

where $C_{b}\left(S_{0}\right)$ is the space of all bounded continuous functions on $S_{0}$.

The measure $m$ could be infinite on a compact neighbourhood of $a$ in $S$, but it is finite on each level set of $u_{\alpha}$ due to the condition A.3. We also note here the next relation which will be utilized in the sequel:

$$
u_{\alpha}(x)=\varphi(x)-\alpha G_{\alpha}^{0} \varphi(x) \leqslant 1-\alpha G_{\alpha}^{0} 1(x), \quad x \in S_{0} .
$$

Define $m_{0}$ by

$$
m_{0}=\varphi \cdot m,
$$

which is an $X^{0}$-excessive measure with $m_{0} p_{t}^{0}=p_{t}^{0} \varphi \cdot m$. In view of Lemma 2.2, there exists a unique $X^{0}$-entrance law $\left\{\mu_{t}\right\}$ related to the measure $m_{0}$ by $(2.22)$, namely,

$$
m_{0}=\int_{0}^{\infty} \mu_{t} \mathrm{~d} t
$$

and it satisfies that

$$
\hat{\mu}_{\alpha}(f)=\left(u_{\alpha}, f\right), \quad f \in \mathcal{B}^{+}\left(S_{0}\right) .
$$

On account of the assumption (A.3), we then have that

$$
\mu_{t}\left(S_{0}\right)<\infty, \quad t>0, \int_{0}^{1} \mu_{t}\left(S_{0}\right) \mathrm{d} t<\infty .
$$

We now introduce the spaces $W^{\prime}, W$ of excursions by

$$
\begin{aligned}
& W^{\prime}=\left\{w: \exists \zeta(w) \in(0, \infty], w \text { is a continuous function from }(0, \zeta(w)) \text { to } S_{0}\right\}, \\
& W=\left\{w \in W^{\prime}: \text { if } \zeta(w)<\infty, \text { then } \exists w(\zeta(w)-) \in\{a\} \cup\{\Delta\}\right\} .
\end{aligned}
$$

$\zeta(w)$ will be called the terminal time of the excursion $w$.

We are concerned with a measure $\mathbf{n}$ on the space $W$ specified in terms of the entrance law $\left\{\mu_{t}\right\}$ and the transition function $p_{t}^{0}$ by

$$
\int_{W} f_{1}\left(w\left(t_{1}\right)\right) f_{2}\left(w\left(t_{2}\right)\right) \cdots f_{n}\left(w\left(t_{n}\right)\right) \mathbf{n}(\mathrm{d} w)=\mu_{t_{1}} f_{1} p_{t_{2}-t_{1}}^{0} f_{2} \cdots p_{t_{n-1}-t_{n-2}}^{0} f_{n-1} p_{t_{n}-t_{n-1}}^{0} f_{n},
$$


for any $0<t_{1}<t_{2}<\cdots<t_{n}, f_{1}, f_{2}, \cdots, f_{n} \in B_{b}\left(S_{0}\right)$. Here, we use the convention that $w \in W$ satisfies $w(t)=\Delta$, $\forall t \geqslant \zeta(w)$, and any function $f$ on $S_{0}$ is extended to $S_{0} \cup \Delta$ by setting $f(\Delta)=0$. Further, on the right-hand side of (4.4), we employ an abbreviated notation for the repeated operations

$$
\mu_{t_{1}}\left[f_{1} p_{t_{2}-t_{1}}^{0}\left\{f_{2} \cdots p_{t_{n-1}-t_{n-2}}^{0}\left(f_{n-1} p_{t_{n}-t_{n-1}}^{0} f_{n}\right)\right\}\right] .
$$

Proposition 4.1. There exists a unique measure $\mathbf{n}$ on the space W satisfying (4.4).

Proof. Let $\mathbf{n}$ be the Kuznetsov measure on $W^{\prime}$ uniquely associated with the transition semigroup $\left\{p_{t}^{0}\right\}$ and the entrance rule $\left\{\eta_{u}\right\}$ defined by

$$
\eta_{u}=0 \quad \text { for } u \leqslant 0, \quad \eta_{u}=\mu_{u} \quad \text { for } u>0
$$

as is constructed in [5, Chapter XIX, 9] for a right semigroup. Because of the present choice of the entrance rule, it holds that $\alpha=0$ where $\alpha$ is the birth time which is random in general (cf. [11, p. 54]).

On account of the assumption A.1 for the diffusion $X^{0}$ on $S_{0}$, the same method of the construction of the Kuznetsov measure as in [5, Chapter XIX, 9] works in proving that $\mathbf{n}$ is supported by the space $W$ and satisfies (4.4).

We call $\mathbf{n}$ the excursion law associated with the entrance law $\left\{\mu_{t}\right\}$. We split the space $W$ of excursions into two parts:

$$
W^{+}=\{w \in W: \zeta(w)<\infty, w(\zeta-)=a\}, \quad W^{-}=W \backslash W^{+} .
$$

Note that $W^{-}=W_{1}^{-} \cup W_{2}^{-}$with

$$
W_{1}^{-}=\{w \in W: \zeta(w)<\infty, w(\zeta-)=\Delta\}, \quad W_{2}^{-}=\{w \in W: \zeta(w)=\infty\} .
$$

For $w \in W^{+}$, we define $\widehat{w} \in W$ by

$$
\widehat{w}(t)=w(\zeta-t), \quad 0<t<\zeta .
$$

The next lemma says that the restriction of the excursion law to $W^{+}$is invariant under time reversion. This is a present variant of the time reversal arguments that have been formulated in general contexts $[22,12,6,7]$.

Lemma 4.1. For any $t_{k}>0$ and $f_{k} \in \mathcal{B}_{b}\left(S_{0}\right)(1 \leqslant k \leqslant n)$,

$$
\begin{aligned}
& \mathbf{n}\left\{\prod_{k=1}^{n} f_{k}\left(w\left(t_{1}+\cdots+t_{k}\right)\right) ; W^{+}\right\}=\mu_{t_{1}} f_{1} p_{t_{2}}^{0} f_{2} \cdots p_{t_{n-1}}^{0} f_{n-1} p_{t_{n}}^{0} f_{n} \varphi, \\
& \mathbf{n}\left\{\prod_{k=1}^{n} f_{k}\left(w\left(t_{1}+\cdots+t_{k}\right)\right) ; W^{+}\right\}=\mathbf{n}\left\{\prod_{k=1}^{n} f_{k}\left(\widehat{w}\left(t_{1}+\cdots+t_{k}\right)\right) ; W^{+}\right\} .
\end{aligned}
$$

Proof. (4.7) readily follows from (4.4) and the Markov property of $\mathbf{n}$. As for (4.8) we observe that, for $\alpha_{1}, \ldots, \alpha_{n}>0$

$$
\int_{0}^{\infty} \cdots \int_{0}^{\infty} \mathrm{e}^{-\alpha_{1} t_{1}-\cdots-\alpha_{n} t_{n}} \mathbf{n}\left\{\prod_{k=1}^{n} f_{k}\left(w\left(t_{1}+\cdots+t_{k}\right)\right) ; W^{+}\right\} \mathrm{d} t_{1} \cdots \mathrm{d} t_{n}
$$

equals

$$
\mathbf{n}\{F(w) ; \zeta<\infty, w(\zeta-)=a\}
$$


with

$$
F(w)=\int \cdots \int_{0<t_{1}<\cdots<t_{n}<\zeta} \prod_{k=1}^{n}\left\{\mathrm{e}^{-\alpha_{k}\left(t_{k}-t_{k-1}\right)} f_{k}\left(w\left(t_{k}\right)\right)\right\} \mathrm{d} t_{1} \cdots \mathrm{d} t_{n} \quad\left(t_{0}=0\right) .
$$

Hence, for (4.8), it suffices to prove

$$
\mathbf{n}\{F(w) ; \zeta<\infty, w(\zeta-)=a\}=\mathbf{n}\{F(\widehat{w}) ; \zeta<\infty, w(\zeta-)=a\} .
$$

Performing the change of variables

$$
\zeta-t_{k}=s_{k}, \quad 1 \leqslant k \leqslant n,
$$

in the expression of $F(\widehat{w})$ and by noting that

$$
\begin{aligned}
& t_{k}=\zeta-s_{k}, \quad t_{k}-t_{k-1}=s_{k-1}-s_{k}, \quad 1 \leqslant k \leqslant n, \quad s_{0}=\zeta, \\
& 0<t_{1}<\cdots<t_{n}<\zeta \quad \Longleftrightarrow 0<s_{n}<\cdots<s_{1}<\zeta,
\end{aligned}
$$

we obtain

$$
\begin{aligned}
F(\widehat{w}) & =\int \cdots \int_{0<s_{n}<\cdots<s_{1}<\zeta} \prod_{k=1}^{n}\left\{\mathrm{e}^{-\alpha_{k}\left(s_{k-1}-s_{k}\right)} f_{k}\left(w\left(s_{k}\right)\right)\right\} \mathrm{d} s_{1} \cdots \mathrm{d} s_{n} \\
& =\int \cdots \int_{0<s_{1}<\cdots<s_{n}<\infty} \Gamma_{s_{1} \cdots s_{n}}(w) \mathrm{d} s_{1} \cdots \mathrm{d} s_{n}
\end{aligned}
$$

with

$$
\Gamma_{s_{1} \cdots s_{n}}(w)=\prod_{k=1}^{n-1}\left\{\mathrm{e}^{-\alpha_{k}\left(s_{k-1}-s_{k}\right)} f_{k}\left(w\left(s_{k}\right)\right)\right\} \cdot \mathrm{e}^{-\alpha_{1}\left(\zeta-s_{1}\right)} I_{(0, \zeta)}\left(s_{1}\right) .
$$

On the other hand, we get from (4.4) and the Markov property of $\mathbf{n}$ that

$$
\begin{aligned}
\mathbf{n}\{ & \left.\Gamma_{s_{1} s_{2} \cdots s_{n}}(w) ; \zeta<\infty, w(\zeta-)=a\right\} \\
= & \mathbf{n}\left\{f_{n}\left(w\left(s_{n}\right)\right) f_{n-1}\left(w\left(s_{n-1}\right)\right) \mathrm{e}^{-\alpha_{n}\left(s_{n-1}-s_{n}\right)} \ldots\right. \\
& f_{2}\left(\left(w\left(s_{2}\right)\right) \mathrm{e}^{-\alpha_{3}\left(s_{2}-s_{3}\right)} f_{1}\left(w\left(s_{1}\right)\right) \mathrm{e}^{-\alpha_{2}\left(s_{1}-s_{2}\right)} u_{\alpha_{1}}\left(w\left(s_{1}\right)\right) ; s_{1}<\zeta\right\} \\
= & \mathrm{e}^{-\alpha_{n}\left(s_{n-1}-s_{n}\right)-\alpha_{n-1}\left(s_{n-2}-s_{n-1}\right)-\cdots-\alpha_{2}\left(s_{1}-s_{2}\right)} \mu_{s_{n}} f_{n} p_{s_{n-1}-s_{n}}^{0} f_{n-1} p_{s_{n-2}-s_{n-1}}^{0} f_{n-1} \cdots p_{s_{2}-s_{3}}^{0} f_{2} p_{s_{1}-s_{2}}^{0} f_{1} u_{\alpha_{1}} .
\end{aligned}
$$

Therefore,

$$
\mathbf{n}\{F(\widehat{w}) ; \zeta<\infty, w(\zeta-)=a\}=\int_{0}^{\infty} \mathrm{d} s_{n} \mu_{s_{n}} f_{n} G_{\alpha_{n}}^{0} f_{n-1} G_{\alpha_{n-1}}^{0} \cdots f_{3} G_{\alpha_{3}}^{0} f_{2} G_{\alpha_{2}}^{0} f_{1} u_{\alpha_{1}} .
$$

In view of (2.7), the symmetry of $G_{\alpha}^{0},(4.7)$ and (4.9), we arrive at

$$
\begin{aligned}
\mathbf{n}\{ & F(\widehat{w}) ; \zeta<\infty, w(\zeta-)=a\}=\left\langle m_{0}, f_{n} G_{\alpha_{n}}^{0} f_{n-1} G_{\alpha_{n-1}}^{0} \cdots f_{3} G_{\alpha_{3}}^{0} f_{2} G_{\alpha_{2}} f_{1} u_{\alpha_{1}}\right\rangle \\
& =\left(f_{n} \varphi, G_{\alpha_{n}}^{0} f_{n-1} G_{\alpha_{n-1}}^{0} \cdots f_{3} G_{\alpha_{3}}^{0} f_{2} G_{\alpha_{2}} f_{1} u_{\alpha_{1}}\right)=\left(f_{1} G_{\alpha_{2}}^{0} f_{2} G_{\alpha_{3}}^{0} f_{3} \cdots G_{\alpha_{n}} f_{n} \varphi, u_{\alpha_{1}}\right) \\
& =\int_{0}^{\infty} \mathrm{e}^{-\alpha_{1} t_{1}} \mu_{t_{1}} f_{1} G_{\alpha_{2}}^{0} f_{2} G_{\alpha_{3}}^{0} f_{3} \cdots G_{\alpha_{n}}^{0} f_{n} \varphi \mathrm{d} t_{1}=\mathbf{n}\{F(w) ; \zeta<\infty, w(\zeta-)=a\}
\end{aligned}
$$

the desired identity (4.10). 
Next we put

$$
W_{a}=\left\{w \in W: \lim _{t \downarrow 0} w(t)=a\right\} .
$$

Lemma 4.2. $\mathbf{n}\left\{W \backslash W_{a}\right\}=0$.

Proof. The preceding lemma implies that

$$
\mathbf{n}\left\{W^{+} \backslash W_{a}\right\}=\mathbf{n}\left\{W^{+} \cap(w(0+)=a)^{c}\right\}=\mathbf{n}\left\{W^{+} \cap(\widehat{w}(0+)=a)^{c}\right\}=\mathbf{n}\left\{W^{+} \cap(w(\zeta-)=a)^{c}\right\}=0 .
$$

We then have for each $t>0$

$$
\mathbf{n}\left\{\varphi(w(t)) ;(\zeta>t) \cap(w(0+)=a)^{c}\right\}=\mathbf{n}\left\{\left(W^{+} \backslash W_{a}\right) \cap(\zeta>t)\right\}=0,
$$

which combined with the assumption A.2 leads us to

$$
\mathbf{n}\left\{\left(W \backslash W_{a}\right) \cap(\zeta>t)\right\}=0 .
$$

It then suffices to let $t \downarrow 0$.

Lemma 4.3. For any neighbourhood $U$ of a in $S$, we let

$$
\tau_{U^{c}}=\inf \left\{t>0: w(t) \in U^{c}\right\}, \quad w \in W .
$$

It holds then that

$$
\mathbf{n}\left\{\tau_{U^{c}}<\zeta\right\}<\infty \text {. }
$$

Proof. We may assume that the closure $\bar{U}$ in $S$ is compact. Let $f(x)=\varphi(x)-u_{1}(x), x \in S_{0}$. Then

$$
f(x)=E_{x}^{0}\left\{1-\mathrm{e}^{-\zeta^{0}} ; \zeta^{0}<\infty, X_{\zeta^{0}-}=a\right\}>0, \quad \forall x \in S_{0} .
$$

Since $u_{\alpha}(x)-u_{1}(x) \uparrow f(x), \alpha \downarrow 0$, the assumption A.3 implies that $f$ is lower semicontinuous on $S_{0}$ and hence

$$
c=\inf _{x \in \partial U} f(x)
$$

is positive. We then have, for each $\delta>0$ and $x \in \partial U$,

$$
\begin{aligned}
P_{x}^{0}\left(\delta<\zeta^{0}<\infty, X_{\zeta^{0}-}=a\right) & \geqslant E_{x}^{0}\left\{1-\mathrm{e}^{-\zeta^{0}} ; \delta<\zeta^{0}<\infty, X_{\zeta^{0}-}=a\right\} \\
& \geqslant c-E_{x}^{0}\left\{1-\mathrm{e}^{-\zeta^{0}} ; \zeta^{0} \leqslant \delta, X_{\zeta^{0}-}=a\right\} \geqslant c-\left(1-\mathrm{e}^{-\delta}\right) .
\end{aligned}
$$

Choose $\delta>0$ so small that

$$
r=c-\left(1-\mathrm{e}^{-\delta}\right)
$$

is positive. For such $\delta$,

$$
P_{x}^{0}\left(\delta<\zeta^{0}<\infty, X_{\zeta^{0}-}=a\right) \geqslant r, \quad \forall x \in \partial U .
$$

We shall use the notation $\tau_{U^{c}}$ not only for $w \in W$ but also for the sample path of the Markov process $X^{0}$. Using the preceding lemma, (4.12) and (4.2), we are led to

$$
\begin{aligned}
\mathbf{n}\left\{\tau_{U^{c}}<\zeta\right\} & =\lim _{\epsilon \downarrow 0} \mathbf{n}\left\{\epsilon<\tau_{U^{c}}<\zeta\right\}=\lim _{\epsilon \downarrow 0} \int_{U} \mu_{\epsilon}(\mathrm{d} x) P_{x}^{0}\left\{\tau_{U^{c}}<\zeta^{0}\right\} \\
& \leqslant \varlimsup_{\epsilon \downarrow} \int_{U} \mu_{\epsilon}(\mathrm{d} x) E_{x}^{0}\left\{r^{-1} P_{X_{\tau_{U^{c}}}^{0}}^{0}\left(\delta<\zeta^{0}<\infty, X_{\zeta^{0}-}=a\right) ; \tau_{U^{c}}<\zeta^{0}\right\}
\end{aligned}
$$




$$
\begin{aligned}
& \leqslant r^{-1} \lim _{\epsilon \downarrow 0} \int_{S_{0}} \mu_{\epsilon}(\mathrm{d} x) P_{x}^{0}\left(\delta<\zeta^{0}<\infty, X_{\zeta^{0}-}=a\right) \leqslant r^{-1} \lim _{\epsilon \downarrow 0} \int_{S_{0}} \mu_{\epsilon}(\mathrm{d} x) P_{x}^{0}\left(\delta<\zeta^{0}\right) \\
& =r^{-1} \lim _{\epsilon \downarrow 0} \mu_{\epsilon+\delta}\left(S_{0}\right) \leqslant r^{-1} \mu_{\delta}\left(S_{0}\right)<\infty .
\end{aligned}
$$

The next lemma states a relation of the excursion law $\mathbf{n}$ to energy functionals $L\left(m_{0}, v\right)$ introduced in Lemma 2.1.

\section{Lemma 4.4.}

(i) $\mathbf{n}\left(W^{+}\right)=L\left(m_{0}, \varphi\right), \mathbf{n}\left(W^{-}\right)=L\left(m_{0}, \psi\right), \mathbf{n}\left(W_{i}^{-}\right)=L\left(m_{0}, \psi^{(i)}\right), i=1,2$.

(ii) $\mathbf{n}\left(W_{1}^{-}\right)<\infty, \mathbf{n}\left(W_{2}^{-}\right)=\mu_{t}\left(\psi^{(2)}\right)=\alpha \hat{\mu}_{\alpha}\left(\psi^{(2)}\right)=\alpha\left(u_{\alpha}, \psi^{(2)}\right)<\infty, t>0, \alpha>0$.

Proof. (i) Since $\mathbf{n}\left(\zeta>t ; W^{+}\right)=\left\langle\mu_{t}, \varphi\right\rangle$, the first identity follows from Lemma 2.2 (v) by letting $t \downarrow 0$. The proof of the other identities is the same.

(ii) Take a neighbourhood $U$ of $a$ in $S$ with compact $\bar{U}$. We have then by the preceding lemma

$$
\mathbf{n}\left(W_{1}^{-}\right)=\mathbf{n}(\zeta<\infty, w(\zeta-)=\Delta) \leqslant \mathbf{n}\left\{\tau_{U^{c}}<\zeta\right\}<\infty .
$$

Since $\psi^{(2)}$ is $p_{t}^{0}$-invariant, the second assertion follows from (i), Lemmas 2.1, 2.2 and assumption A.3.

In particular, $\mathbf{n}\left(W^{-}\right)=\mathbf{n}\left(W_{1}^{-}\right)+\mathbf{n}\left(W_{2}^{-}\right)$is finite. We shall see that $\mathbf{n}\left(W^{+}\right)=\infty$.

\subsection{Poisson point processes on $W_{a}$ and a new process $X$}

By Lemma 4.2, the excursion law $\mathbf{n}$ is concentrated on the space $W_{a}$ defined by (4.11). Accordingly, we consider the spaces

$$
W_{a}^{+}=\left\{w \in W^{+}: \lim _{t \downarrow 0} w(t)=a\right\}, \quad W_{a}^{-}=\left\{w \in W^{-}: \lim _{t \downarrow 0} w(t)=a\right\},
$$

so that $W_{a}=W_{a}^{+}+W_{a}^{-}$. In the sequel however, we shall employ slightly modified but equivalent definitions of those spaces by extending each $w$ from an $S_{0}$-valued excursion to $S$-valued continuous one as follows:

$$
\begin{gathered}
W_{a}=\{w: \exists \zeta(w) \in(0, \infty], w \text { is a continuous function from }[0, \zeta(w)) \text { to } S, w(0)=a . \\
\left.w(t) \in S_{0}, t \in(0, \zeta(w)), w(\zeta(w)-) \in\{a\} \cup\{\Delta\} \text { if } \zeta(w)<\infty\right\} .
\end{gathered}
$$

Any $w \in W_{a}$ for which $\zeta(w)<\infty, w(\zeta(w)-)=a$ will be regarded to be a continuous function from $[0, \zeta(w)]$ to $S$ by setting $w(\zeta(w))=a$. We further let

$$
\begin{gathered}
W_{a}^{+}=\{w: \exists \zeta(w) \in(0, \infty), w \text { is a continuous function from }[0, \zeta(w)] \text { to } S, \\
\left.w(t) \in S_{0}, t \in(0, \zeta(w)), w(0)=w(\zeta(w))=a\right\}, \\
W_{a}^{-}=W_{a} \backslash W_{a}^{+} .
\end{gathered}
$$

The excursion law $\mathbf{n}$ will be considered to be a measure on $W_{a}$ defined by (4.13) and we denote by $\mathbf{n}^{+}, \mathbf{n}^{-}$, the restrictions of $\mathbf{n}$ to $W_{a}^{+}, W_{a}^{-}$defined by (4.14) and (4.15) respectively.

Let $\left\{\mathbf{p}_{s}, s>0\right\}$ be a Poisson point process on $W_{a}$ with characteristic measure $\mathbf{n}$ defined on an appropriate probability space $(\Omega, P)$. We then let

$$
\begin{aligned}
& \mathbf{p}_{s}^{+}= \begin{cases}\mathbf{p}_{s} & \text { if } \mathbf{p}_{s} \in W_{a}^{+}, \\
\partial & \text { otherwise, }\end{cases} \\
& \mathbf{p}_{s}^{-}= \begin{cases}\mathbf{p}_{s} & \text { if } \mathbf{p}_{s} \in W_{a}^{-}, \\
\partial & \text { otherwise, }\end{cases}
\end{aligned}
$$


where $\partial$ is an extra point disjoint of $W_{a}$. Then $\left\{\mathbf{p}_{s}^{+}, s>0\right\},\left\{\mathbf{p}_{s}^{-}, s>0\right\}$ are mutually independent Poisson point processes on $W_{a}^{+}, W_{a}^{-}$with characteristic measures $\mathbf{n}^{+}, \mathbf{n}^{-}$respectively. Furthermore

$$
\mathbf{p}_{s}=\mathbf{p}_{s}^{+}+\mathbf{p}_{s}^{-} \text {. }
$$

By means of the terminal time $\zeta\left(\mathbf{p}_{r}^{+}\right)$of the excursion $\mathbf{p}_{r}^{+}$, we let

$$
J(s)=\sum_{r \leqslant s} \zeta\left(\mathbf{p}_{r}^{+}\right), \quad s>0 .
$$

We put $J(0)=0$.

\section{Lemma 4.5.}

(i) $J(s)<\infty$ a.s. for $s>0$.

(ii) $\{J(s)\}_{s \geqslant 0}$ is a subordinator with

$$
E\left\{\mathrm{e}^{-\alpha J(s)}\right\}=\exp \left\{-\alpha\left(u_{\alpha}, \varphi\right) s\right\} .
$$

Proof. (i) We write $J(s)$ as $J(s)=I+I I$ with

$$
I=\sum_{r \leqslant s, \zeta\left(\mathbf{p}_{r}^{+}\right) \leqslant 1} \zeta\left(\mathbf{p}_{r}^{+}\right), \quad I I=\sum_{r \leqslant s, \zeta\left(\mathbf{p}_{r}^{+}\right)>1} \zeta\left(\mathbf{p}_{r}^{+}\right) .
$$

Since $\mathbf{n}^{+}(\zeta>1) \leqslant \mu_{1}\left(S_{0}\right)<\infty$ by (4.2), $r$ in the sum $I I$ is finite a.s. and hence $I I<\infty$ a.s. On the other hand,

$$
E(I)=s \mathbf{n}^{+}(\zeta ; \zeta \leqslant 1) \leqslant s \mathbf{n}^{+}(\zeta \wedge 1)=s \mathbf{n}^{+}\left\{\int_{0}^{1} I_{(0, \zeta)}(t) \mathrm{d} t\right\}=s \int_{0}^{1} \mathbf{n}^{+}(\zeta>t) \mathrm{d} t \leqslant s \int_{0}^{1} \mu_{t}\left(S_{0}\right) \mathrm{d} t,
$$

which is finite by (4.2). Hence $I<\infty$ a.s.

(ii) Clearly $\{J(s)\}_{s \geqslant 0}$ is increasing and of stationary independent increment. Since

$$
\mathrm{e}^{-\alpha J(s)}=\sum_{r \leqslant s}\left\{\mathrm{e}^{-\alpha J(r)}-\mathrm{e}^{-\alpha J(r-)}\right\}=\sum_{r \leqslant s} \mathrm{e}^{-\alpha J(r-)}\left\{\mathrm{e}^{-\alpha \zeta\left(\mathbf{p}_{r}^{+}\right)}-1\right\},
$$

we have

$$
E\left\{\mathrm{e}^{-\alpha J(s)}\right\}=-c \int_{0}^{s} E\left\{\mathrm{e}^{-\alpha J(r)}\right\} \mathrm{d} r
$$

with

$$
\begin{aligned}
c & =\mathbf{n}^{+}\left(1-\mathrm{e}^{-\alpha \zeta}\right)=\mathbf{n}\left(1-\mathrm{e}^{-\alpha \zeta} ; \zeta<\infty, w(\zeta)=a\right)=\mathbf{n}\left\{\alpha \int_{0}^{\zeta} \mathrm{e}^{-\alpha t} \mathrm{~d} t ; \zeta<\infty, w(\zeta)=a\right\} \\
& =\alpha \int_{0}^{\infty} \mathrm{e}^{-\alpha t} \mathbf{n}(t<\zeta<\infty, w(\zeta)=a) \mathrm{d} t=\alpha \int_{0}^{\infty} \mathrm{e}^{-\alpha t} \mu_{t}(\varphi) \mathrm{d} t=\alpha \hat{\mu}_{\alpha}(\varphi)=\alpha\left(u_{\alpha}, \varphi\right)<\infty .
\end{aligned}
$$

In virtue of Lemmas 4.3 and 4.5, we may assume that the next three properties hold for any $\omega \in \Omega$ by subtracting a $P$-negligible set from $\Omega$ if necessary: 


$$
\begin{aligned}
& J(s)<\infty \quad \forall s>0, \\
& \lim _{s \rightarrow \infty} J(s)=\infty,
\end{aligned}
$$

and, for any finite interval $I \subset(0, \infty)$ and any neighbourhood $U$ of $a$ in $S$,

$$
\left\{s \in I: \tau_{U^{c}}\left(\mathbf{p}_{s}^{+}\right)<\zeta\left(\mathbf{p}_{s}^{+}\right)\right\} \quad \text { is a finite set. }
$$

Let $T$ be the time of occurrence of the first excursion of the point process $\left\{\mathbf{p}_{s}^{-}, s>0\right\}$, namely,

$$
T=\min \left\{s>0: \mathbf{p}_{s}^{-} \neq \partial\right\} .
$$

Since $\mathbf{n}\left(W_{a}^{-}\right)=L\left(m_{0}, \psi\right)<\infty$ by Lemma 4.4, we can see that $T$ and $\mathbf{p}_{T}^{-}$are independent and

$$
P(T>t)=\mathrm{e}^{-L\left(m_{0}, \psi\right) t}, \quad \text { the distribution of } \mathbf{p}_{T}^{-}=L\left(m_{0}, \psi\right)^{-1} \mathbf{n}^{-} .
$$

We are now in a position to produce a new process $X=\left\{X_{t}\right\}_{t \geqslant 0}$ out of the point processes of excursions $\mathbf{p}^{ \pm}$.

(i) For $0 \leqslant t<J(T-)$, we determine $s$ by

$$
J(s-) \leqslant t \leqslant J(s),
$$

and let

$$
X_{t}= \begin{cases}\mathbf{p}_{s}^{+}(t-J(s-)) & \text { if } J(s)-J(s-)>0, \\ a & \text { if } J(s)-J(s-)=0 .\end{cases}
$$

(ii) For $J(T-) \leqslant t<\zeta_{\omega} \equiv J(T-)+\zeta\left(\mathbf{p}_{T}^{-}\right)$, we let

$$
X_{t}=\mathbf{p}_{T}^{-}(t-J(T-)) \text {. }
$$

In this way, the $S$-valued continuous path

$$
X_{t}, \quad 0 \leqslant t<\zeta_{\omega},
$$

is defined and

$$
X_{\zeta^{-}}=\Delta \text { if } \zeta_{\omega}<\infty .
$$

Continuity of the path is a consequence of (4.23).

For this process $\left\{X_{t}, 0 \leqslant t<\zeta_{\omega}, P\right\}$, let us put

$$
G_{\alpha} f(a)=E\left(\int_{0}^{\zeta_{\omega}} \mathrm{e}^{-\alpha t} f\left(X_{t}\right) \mathrm{d} t\right), \quad \alpha>0, f \in \mathcal{B}(S) .
$$

Proposition 4.2. It holds that

$$
G_{\alpha} f(a)=\frac{\left(u_{\alpha}, f\right)}{\alpha\left(u_{\alpha}, \varphi\right)+L\left(m_{0}, \psi\right)} .
$$

Proof. We use the notation

$$
\hat{f}_{\alpha}(w)=\int_{0}^{\zeta(w)} \mathrm{e}^{-\alpha t} f(w(t)) \mathrm{d} t, \quad w \in W_{a} .
$$

We have then 


$$
\begin{aligned}
\int_{0}^{\zeta_{\omega}} \mathrm{e}^{-\alpha t} f\left(X_{t}\right) \mathrm{d} t & =\sum_{s<T} \int_{J(s-)}^{J(s)} \mathrm{e}^{-\alpha t} f\left(X_{t}\right) \mathrm{d} t+\int_{J(T-)}^{J(T-)+\zeta\left(\mathbf{p}_{T}^{-}\right)} \mathrm{e}^{-\alpha t} f\left(X_{t}\right) \mathrm{d} t \\
& =\sum_{s<T} \mathrm{e}^{-\alpha J(s-)} \hat{f}_{\alpha}\left(\mathbf{p}_{s}^{+}\right)+\mathrm{e}^{-\alpha J(T-)} \hat{f}_{\alpha}\left(\mathbf{p}_{T}^{-}\right),
\end{aligned}
$$

and consequently

$$
\begin{aligned}
G_{\alpha} f(a) & =E\left(\sum_{s<T} \mathrm{e}^{-\alpha J(s-)} \hat{f}_{\alpha}\left(\mathbf{p}_{s}^{+}\right)+\mathrm{e}^{-\alpha J(T-)} \hat{f}_{\alpha}\left(\mathbf{p}_{T}^{-}\right)\right) \\
& =E\left(\int_{0}^{T} \mathrm{e}^{-\alpha \hat{\mu}_{\alpha}(\varphi) s} \mathrm{~d} s\right) \mathbf{n}^{+}\left(\hat{f}_{\alpha}\right)+E\left(\mathrm{e}^{-\alpha \hat{\mu}_{\alpha}(\varphi) T}\right) L\left(m_{0}, \psi\right)^{-1} \mathbf{n}^{-}\left(\hat{f}_{\alpha}\right) \\
& =\frac{\mathbf{n}^{+}\left(\hat{f}_{\alpha}\right)}{\alpha \hat{\mu}_{\alpha}(\varphi)+L\left(m_{0}, \psi\right)}+\frac{\mathbf{n}^{-}\left(\hat{f}_{\alpha}\right)}{\alpha \hat{\mu}_{\alpha}(\varphi)+L\left(m_{0}, \psi\right)} \\
& =\frac{\mathbf{n}\left(\hat{f}_{\alpha}\right)}{\alpha \hat{\mu}_{\alpha}(\varphi)+L\left(m_{0}, \psi\right)}=\frac{\hat{\mu}_{\alpha}(f)}{\alpha \hat{\mu}_{\alpha}(\varphi)+L\left(m_{0}, \psi\right)}
\end{aligned}
$$

It then suffices to substitute (4.1) in the last expression.

\subsection{Continuity of resolvent along $X$}

Lemma 4.6. For $\alpha>0$ and $f \in \mathcal{B}(S)$, define $G_{\alpha} f(a)$ by the right-hand side of (4.30) and extend it to a function on $S$ by setting

$$
G_{\alpha} f(x)=G_{\alpha}^{0} f(x)+G_{\alpha} f(a) u_{\alpha}(x), \quad x \in S_{0} .
$$

Then $\left\{G_{\alpha}\right\}_{\alpha>0}$ is an m-symmetric (sub)Markovian resolvent on $S$.

Proof. By making use of the resolvent equation for $G_{\alpha}^{0}$, the $m$-symmetry of $G_{\alpha}^{0}$ and the equation

$$
u_{\alpha}(x)-u_{\beta}(x)+(\alpha-\beta) G_{\alpha}^{0} u_{\beta}(x)=0, \quad \alpha, \beta>0, x \in S_{0},
$$

we can easily check the resolvent equation

$$
G_{\alpha} f(x)-G_{\beta} f(x)+(\alpha-\beta) G_{\alpha} G_{\beta} f(x)=0, \quad x \in S .
$$

The $m$-symmetry of $G_{\alpha}$

$$
\int_{S} G_{\alpha} f(x) g(x) m(\mathrm{~d} x)=\int_{S} f(x) G_{\alpha} g(x) m(\mathrm{~d} x)
$$

holding for any non-negative Borel functions $f, g$ is clear. Moreover we get by Lemma 2.1 that

$$
\begin{aligned}
\alpha G_{\alpha} 1(x) & =\alpha G_{\alpha}^{0} 1(x)+u_{\alpha}(x) \frac{\alpha\left(u_{\alpha}, \varphi+\psi\right)}{\alpha\left(u_{\alpha}, \varphi\right)+L\left(m_{0}, \psi\right)} \\
& \leqslant 1-u_{\alpha}(x)+u_{\alpha}(x)=1, \quad x \in S_{0},
\end{aligned}
$$

and similarly, $\alpha G_{\alpha} 1(a) \leqslant 1$. 
Let $\left\{U_{n}\right\}$ be a decreasing sequence of open neighbourhoods of the point $a$ in $S$ such that $U_{n} \supset \bar{U}_{n+1}$ and $\bigcap_{n=1}^{\infty} U_{n}=\{a\}$. Let

$$
A=A_{\alpha, \rho}=\left\{x \in S_{0}: u_{\alpha}(x)<\rho\right\} \text { for } \alpha>0,0<\rho<1 .
$$

We then set

$$
\sigma_{n}=\inf \left\{t>0: X_{t}^{0} \in U_{n} \cap S_{0}\right\}, \quad \sigma_{a}=\lim _{n \rightarrow \infty} \sigma_{n}, \quad \tau_{n}=\inf \left\{t>0: X_{t}^{0} \in U_{n} \cap A\right\},
$$

with the convention that $\inf \emptyset=\infty$.

Lemma 4.7. For any $\alpha>0, \rho \in(0,1)$ and $x \in S_{0}$,

$$
\lim _{n \rightarrow \infty} P_{x}^{0}\left\{\tau_{n}<\sigma_{a}<\infty\right\}=0
$$

Proof. Since

$$
\left\{\sigma_{a}<\infty\right\}=\left\{\zeta^{0}<\infty, X_{\zeta^{0}-}^{0}=a\right\}
$$

and $\sigma_{a}=\zeta^{0}$ on the set $\left\{\sigma_{a}<\infty\right\}$, we have for $x \in S_{0}$ and $m<n$

$$
\begin{aligned}
u_{\alpha}(x) & =E_{x}^{0}\left\{\mathrm{e}^{-\alpha \sigma_{a}} ; \tau_{n}<\sigma_{a}\right\}+E_{x}^{0}\left\{\mathrm{e}^{-\alpha \sigma_{a}} ; \tau_{n} \geqslant \sigma_{a}\right\}=E_{x}^{0}\left\{\mathrm{e}^{-\alpha \tau_{n}} u_{\alpha}\left(X_{\tau_{n}}^{0}\right) ; \tau_{n}<\sigma_{a}\right\}+E_{x}^{0}\left\{\mathrm{e}^{-\alpha \sigma_{a}} ; \tau_{n} \geqslant \sigma_{a}\right\} \\
& \leqslant \rho E_{x}^{0}\left\{\mathrm{e}^{-\alpha \tau_{n}} ; \tau_{n}<\sigma_{a}\right\}+E_{x}^{0}\left\{\mathrm{e}^{-\alpha \sigma_{a}} ; \tau_{n} \geqslant \sigma_{a}\right\} \leqslant \rho E_{x}^{0}\left\{\mathrm{e}^{-\alpha\left(\tau_{n} \wedge \sigma_{a}\right)} ; \tau_{m}<\sigma_{a}\right\}+E_{x}^{0}\left\{\mathrm{e}^{-\alpha \sigma_{a}} ; \tau_{n} \geqslant \sigma_{a}\right\} .
\end{aligned}
$$

By letting first $n \rightarrow \infty$ and then $m \rightarrow \infty$, we obtain

$$
\begin{aligned}
u_{\alpha}(x) & \leqslant \rho \lim _{m \rightarrow \infty} E_{x}^{0}\left\{\mathrm{e}^{-\alpha \sigma_{a}} ; \tau_{m}<\sigma_{a}\right\}+\lim _{n \rightarrow \infty} E_{x}^{0}\left\{\mathrm{e}^{-\alpha \sigma_{a}} ; \tau_{n} \geqslant \sigma_{a}\right\} \\
& =E_{x}^{0}\left\{\mathrm{e}^{-\alpha \sigma_{a}}\right\}-(1-\rho) \lim _{n \rightarrow \infty} E_{x}^{0}\left\{\mathrm{e}^{-\alpha \sigma_{a}} ; \tau_{n}<\sigma_{a}\right\} \\
& =u_{\alpha}(x)-(1-\rho) \lim _{n \rightarrow \infty} E_{x}^{0}\left\{\mathrm{e}^{-\alpha \sigma_{a}} ; \tau_{n}<\sigma_{a}\right\},
\end{aligned}
$$

which implies

$$
\lim _{n \rightarrow \infty} E_{x}^{0}\left\{\mathrm{e}^{-\alpha \sigma_{a}} ; \tau_{n}<\sigma_{a}\right\}=0
$$

and so (4.32) must hold.

Lemma 4.8. Let $\alpha>0$.

(i) For any $x \in S_{0}$,

$$
\lim _{t \uparrow \sigma_{a}} u_{\alpha}\left(X_{t}^{0}\right)=1 \quad P_{x}^{0} \text {-a.s. on }\left\{\sigma_{a}<\infty\right\} .
$$

(ii) $\mathbf{n}(\Lambda)=0$ where

$$
\Lambda=\left\{w \in W_{a}^{+}: \exists \alpha>0, \lim _{t \uparrow \zeta} u_{\alpha}(w(t)) \neq 1\right\} .
$$

Proof. If $\sigma_{a}<\infty$ and if $\underline{\lim }_{t \uparrow \sigma_{a}} u_{\alpha}\left(X_{t}^{0}\right)<\rho$, then for any small $\epsilon>0$ there exists $t \in\left(\sigma_{a}-\epsilon, \sigma_{a}\right)$ such that $u_{\alpha}\left(X_{t}^{0}\right)<\rho$, and so $\tau_{n}<\sigma_{a}$ for all $n$. Therefore by the preceding lemma

$$
P_{x}^{0}\left\{\frac{\lim }{t \uparrow \sigma_{a}} u_{\alpha}\left(X_{t}^{0}\right)<\rho, \sigma_{a}<\infty\right\}=0 .
$$

Since $u_{\alpha}$ is decreasing in $\alpha$ and $\rho$ can be taken arbitrarily close to 1 , we obtain (4.33). 
(ii) follows from (i) as

$$
\mathbf{n}(\Lambda)=\lim _{\epsilon \downarrow 0} \mathbf{n}(\Lambda \cap\{\epsilon<\zeta\})=\lim _{\epsilon \downarrow 0} \int_{S_{0}} \mu_{\epsilon}(\mathrm{d} x) P_{x}^{0}\left(\lim _{t \uparrow \sigma_{a}} u_{\alpha}\left(X_{t}^{0}\right) \neq 1\right)=0 .
$$

We extend $u_{\alpha}$ to a function on $S$ by setting $u_{\alpha}(a)=1$. By Lemma 4.8(ii) combined with Lemma 4.1 and a similar reasoning as in the proof of Lemma 4.2, we may assume, subtracting a suitable n-negligible set from $W_{a}^{+}$ (resp. $W_{a}^{-}$), that $u_{1}(w(t))$ is continuous in $t \in[0, \zeta]($ resp. $t \in[0, \zeta)$ ).

Lemma 4.9. Let $0<\rho<1$ and set

$$
\widetilde{W}_{\rho}=\left\{w \in W_{a}^{+}: \max _{0 \leqslant t \leqslant \zeta}\left\{1-u_{1}(w(t))\right\}>\rho\right\} .
$$

Then $\mathbf{n}^{+}\left(\widetilde{W}_{\rho}\right)<\infty$.

Proof. The proof is similar to that of Lemma 4.3. For any $x$ such that $1-u_{1}(x) \geqslant \rho$ and for $\delta=-\log \left(1-\frac{\rho}{2}\right)>0$, we have

$$
\begin{aligned}
P_{x}^{0}\left(\sigma_{a}>\delta\right) & \geqslant E_{x}^{0}\left\{1-\mathrm{e}^{-\sigma_{a}} ; \sigma_{a}>\delta\right\}=E_{x}^{0}\left\{1-\mathrm{e}^{-\sigma_{a}}\right\}-E_{x}^{0}\left\{1-\mathrm{e}^{-\sigma_{a}} ; \sigma_{a} \leqslant \delta\right\} \\
& \geqslant 1-u_{1}(x)-\left(1-\mathrm{e}^{-\delta}\right) \geqslant \rho-\left(1-\mathrm{e}^{-\delta}\right)=\frac{\rho}{2} .
\end{aligned}
$$

Therefore if we set

$$
A=\left\{x \in S_{0}: 1-u_{1}(x) \leqslant \rho\right\}, \quad \tau=\inf \left\{t>0: w(t) \in S_{0} \backslash A\right\},
$$

then

$$
\begin{aligned}
\mathbf{n}^{+}\left(\tilde{W}_{\rho}\right) & =\mathbf{n}^{+}(\tau<\zeta)=\lim _{\epsilon \downarrow 0} \mathbf{n}^{+}\left(\epsilon<\tau<\zeta^{0}\right)=\lim _{\epsilon \downarrow 0} \int_{A} \mu_{\epsilon}(\mathrm{d} x) P_{x}^{0}\left(\tau<\zeta^{0}\right) \\
& \leqslant \varlimsup_{\epsilon \downarrow 0} \int_{A} \mu_{\epsilon}(\mathrm{d} x) E_{x}^{0}\left\{\left(\frac{2}{\rho}\right) P_{X_{\tau}^{0}}^{0}\left(\sigma_{a}>\delta\right) ; \tau<\zeta^{0}\right\} \leqslant \frac{2}{\rho} \varlimsup_{\epsilon \downarrow 0} \int_{S_{0}} \mu_{\epsilon}(\mathrm{d} x) P_{x}^{0}\left(\sigma_{a}>\delta\right) \\
& \leqslant \frac{2}{\rho} \lim _{\epsilon \downarrow 0} \int_{S_{0}} \mu_{\epsilon}(\mathrm{d} x) P_{x}^{0}\left(\zeta^{0}>\delta\right)+\frac{2}{\rho} \lim _{\epsilon \downarrow 0} \int_{S_{0}} \mu_{\epsilon}(\mathrm{d} x) P_{x}^{0}\left(\zeta^{0}<\sigma_{a}=\infty\right) \\
& =\frac{2}{\rho} \lim _{\epsilon \downarrow 0} \mu_{\epsilon+\delta}(1)+\frac{2}{\rho} \lim _{\epsilon \downarrow 0} \mu_{\epsilon}\left(\psi^{(1)}\right),
\end{aligned}
$$

which is finite in view of (4.2) and Lemma 4.4.

For $\alpha>0, f \in \mathcal{B}(S)$, we defined the resolvent $G_{\alpha} f$ by

$$
G_{\alpha} f(x)=G_{\alpha}^{0} f(x)+G_{\alpha} f(a) u_{\alpha}(x), \quad x \in S_{0},
$$

with $G_{\alpha} f(a)$ of Proposition 4.2. We now extend $G_{\alpha}^{0} f(x)$ to $S$ by setting

$$
G_{\alpha}^{0} f(a)=0 .
$$

In the last subsection, we have constructed a process $\left\{X_{t}\right\}_{t \in\left[0, \zeta_{\omega}\right)}$ out of the Poisson point processes $\mathbf{p}^{+}, \mathbf{p}^{-}$on $W_{a}^{+}, W_{a}^{-}$defined on a probability space $(\Omega, P)$.

Proposition 4.3. Let $u=G_{\alpha} f$ with $f \in C_{b}(S)$. Then $u\left(X_{t}\right)$ is continuous in $t \in\left[0, \zeta_{\omega}\right), P$-a.s. 
Proof. As was remarked immediately after the proof of Lemma 4.8, $u_{1}$ is continuous along any sample point functions of $\mathbf{p}^{+}=\left\{\mathbf{p}_{s}^{+}, s>0\right\}$ and $\mathbf{p}^{-}=\left\{\mathbf{p}_{s}^{-}, s>0\right\}$. Moreover, by Lemma 4.9, we can subtract a suitable $P$ negligible set from $\Omega$ so that, in addition to the properties (4.21), (4.22) and (4.23), $\mathbf{p}^{+}$satisfies the following property for every sample point $\omega \in \Omega$ : for any finite interval $I \subset(0, \infty)$ and for any $\rho \in(0,1)$,

$$
\left\{s \in I: \max _{0 \leqslant t \leqslant \zeta\left(\mathbf{p}_{s}^{+}\right)}\left(1-u_{1}\left(\mathbf{p}_{s}^{+}(t)\right)\right)>\rho\right\} \text { is a finite set. }
$$

Then it is not hard to see that not only $X_{t}$ but also $u_{1}\left(X_{t}\right)$ are continuous in $t \in\left[0, \zeta_{\omega}\right)$. From the inequality $G_{1}^{0} 1(x) \leqslant 1-u_{1}(x), x \in S$, we see that

$$
\lim _{t \rightarrow t_{0}} G_{1}^{0} 1\left(X_{t}\right)=0 \quad \text { if } X_{t_{0}}=a .
$$

Hence $G_{1}^{0} f\left(X_{t}\right)$ has the same property as the above for $f \in C_{b}(S)$. Since $G_{1}^{0} f\left(X_{t}\right)$ is clearly continuous on $\left\{t \in\left[0, \zeta_{\omega}\right): X_{t} \neq a\right\}$ by the assumption A.4, it is continuous on $\left[0, \zeta_{\omega}\right)$. We have thus proved the continuity of $G_{1} f\left(X_{t}\right)$. The continuity of $G_{\alpha} f\left(X_{t}\right)$ follows from the resolvent equation proved in Lemma 4.6.

\subsection{Markov property of $X$}

Let us define $p_{t} f(x)$ for $t>0, x \in S, f \in \mathcal{B}(S)$, as follows:

$$
\begin{aligned}
& p_{t} f(a)=E\left(f\left(X_{t}\right) ; \zeta_{\omega}>t\right), \\
& p_{t} f(x)=p_{t}^{0} f(x)+E_{x}^{0}\left\{p_{t-\sigma_{a}} f(a) ; \sigma_{a} \leqslant t\right\}, \quad x \in S_{0} .
\end{aligned}
$$

Evidently

$$
\int_{0}^{\infty} \mathrm{e}^{-\alpha t} p_{t} f \mathrm{~d} t=G_{\alpha} f, \quad \alpha>0 .
$$

Lemma 4.10. $p_{t+s}=p_{t} p_{s}, t, s>0$.

Proof. Take any $f \in C_{b}(S)$. By (4.36) and the resolvent equation in Lemma 4.6, we have for any $x \in S$

$$
\int_{0}^{\infty} \mathrm{e}^{-\alpha t}\left\{\int_{0}^{\infty} \mathrm{e}^{-\beta s} p_{t+s} f(x) \mathrm{d} s\right\} \mathrm{d} t=\int_{0}^{\infty} \mathrm{e}^{-\alpha t}\left\{p_{t}\left(G_{\beta} f\right)(x)\right\} \mathrm{d} t,
$$

because the left-hand side equals $\frac{1}{\alpha-\beta}\left(G_{\beta} f(x)-G_{\alpha} f(x)\right)=G_{\alpha} G_{\beta} f(x)$.

We first consider the case where $x=a$. Then the functions inside $\{\cdot\}$ of the both hand sides of (4.38) are continuous in $t>0$ in virtue of the continuity of $X$ and Proposition 4.3. Hence we have for any $t>0$

$$
\int_{0}^{\infty} \mathrm{e}^{-\alpha s} p_{t+s} f(a) \mathrm{d} s=p_{s}\left(G_{\beta} f\right)(a)=\int_{0}^{\infty} \mathrm{e}^{-\beta s} p_{t}\left(p_{s} f\right)(a) \mathrm{d} s .
$$

Since both $p_{t+s} f(a), p_{t}\left(p_{s} f\right)(a)$ are right continuous in $s>0$, we get

$$
p_{t+s} f(a)=p_{t}\left(p_{s} f\right)(a), \quad t>0, s>0 .
$$

We next consider the case where $x \in S_{0}$. Using (4.37), we obtain

$$
\begin{aligned}
p_{t+s} f(x) & =p_{t+s}^{0} f(x)+E_{x}^{0}\left\{p_{t+s-\sigma_{a}} f(a) ; \sigma_{a} \leqslant t+s\right\} \\
& =p_{t+s}^{0} f(x)+E_{x}^{0}\left\{p_{t-\sigma_{a}}\left(p_{s} f\right)(a) ; \sigma_{a} \leqslant t\right\}+E_{x}^{0}\left\{p_{t+s-\sigma_{a}} f(a): t<\sigma_{a} \leqslant t+s\right\} .
\end{aligned}
$$


On the other hand,

$$
p_{t}\left(p_{s} f\right)(x)=p_{t}^{0}\left(p_{s} f\right)(x)+E_{x}^{0}\left\{p_{t-\sigma_{a}}\left(p_{s} f\right)(a) ; \sigma_{a} \leqslant t\right\} .
$$

Hence it suffices to prove that

$$
p_{t+s}^{0} f(x)+E_{x}^{0}\left\{p_{t+s-\sigma_{a}} f(a) ; t<\sigma_{a} \leqslant t+s\right\}=p_{t}^{0}\left(p_{s} f\right)(x) .
$$

Put

$$
g(x)=E_{x}^{0}\left\{p_{s-\sigma_{a}} f(a) ; \sigma_{a} \leqslant s\right\},
$$

then, we are led from $p_{s} f(x)=p_{s}^{0} f(x)+g(x)$ to

$$
p_{t}^{0}\left(p_{s} f\right)(x)=p_{t+s}^{0} f(x)+p_{t}^{0} g(x),
$$

and consequently, (4.40) is reduced to

$$
E_{x}^{0}\left\{p_{t+s-\sigma_{a}} f(a) ; t<\sigma_{a} \leqslant t+s\right\}=E_{x}^{0}\left(g\left(X_{t}^{0}\right) ; \zeta^{0}>t\right) .
$$

With the notation $\theta_{t}$ to denote the usual shift, the left-hand side of (4.41) equals

$$
\begin{aligned}
E_{x}^{0}\left\{p_{t+s-\sigma_{a}} f(a) ; \zeta^{0}>t, \sigma_{a}>t, \sigma_{a} \circ \theta_{t} \leqslant s\right\} & =E_{x}^{0}\left\{p_{s-\sigma_{a} \circ \theta_{t}} f(a) ; \zeta^{0}>t, \sigma_{a} \circ \theta_{t} \leqslant s\right\} \\
& =E_{x}^{0}\left[E_{X_{t}^{0}}^{0}\left\{p_{s-\sigma_{a}} f(a) ; \sigma_{a} \leqslant s\right\} ; \zeta^{0}>t\right],
\end{aligned}
$$

which coincides with the right-hand side of (4.41) as was to be proved.

Lemma 4.11. Suppose $g \in \mathcal{B}(S)$ and $\lim _{\epsilon \downarrow 0} p_{\epsilon} g(x)=g(x), x \in S$. Then, for any $f \in C_{b}(S), t>0$,

$$
\lim _{\epsilon \downarrow 0} p_{\epsilon}\left(f p_{t} g\right)(x)=f(x) p_{t} g(x), \quad x \in S .
$$

Proof. Fix $x \in S$. Clearly, for any neighbourhood $U$ of $x$,

$$
\lim _{\epsilon \downarrow 0} p_{\epsilon} I_{U}(x)=1,
$$

and hence

$$
p_{\epsilon}\left|f p_{t} g\right|(x)=p_{\epsilon}\left|f I_{U} p_{t} g\right|(x)+o(\epsilon) .
$$

For any $\delta>0$, take a neighbourhood $U$ of $x$ such that

$$
|f(y)-f(x)|<\delta, \quad y \in U .
$$

Then

$$
\begin{aligned}
\left|p_{\epsilon}\left(f p_{t} g\right)(x)-f(x) p_{\epsilon}\left(p_{t} g\right)(x)\right| & \leqslant p_{\epsilon}\left(|f-f(x)|\left|p_{t} g\right|\right)(x) \\
& \leqslant p_{\epsilon}\left(|f-f(x)| I_{U}\left|p_{t} g\right|\right)(x)+\mathrm{o}(\epsilon) \leqslant \delta\|g\|_{\infty}+\mathrm{o}(\epsilon) .
\end{aligned}
$$

On the other hand, we have from the preceding lemma that

$$
\lim _{\epsilon \downarrow 0} f(x) p_{\epsilon}\left(p_{t} g\right)(x)=\lim _{\epsilon \downarrow 0} f(x) p_{t}\left(p_{\epsilon} g\right)(x)=f(x) p_{t} g(x) .
$$

Consequently

$$
\varlimsup_{\epsilon \downarrow 0}\left|p_{\epsilon}\left(f p_{t} g\right)(x)-f(x) p_{t} g(x)\right| \leqslant \delta\|g\|_{\infty},
$$

which means (4.45) because $\delta>0$ can be taken arbitrarily small. 


\section{Proposition 4.4.}

(i) For $\alpha_{1}, \ldots, \alpha_{n}>0$,

$$
E\left\{\int_{0<t_{1}<\cdots<t_{n}<\zeta_{\omega}} \cdots \prod_{k=1}^{n}\left(\mathrm{e}^{-\alpha_{k}\left(t_{k}-t_{k-1}\right)} f_{k}\left(X_{t_{k}}\right)\right) \mathrm{d} t_{1} \cdots \mathrm{d} t_{n}\right\}=G_{\alpha_{1}} f_{1} G_{\alpha_{2}} f_{2} \cdots G_{\alpha_{n}} f_{n}(a),
$$

where we set $t_{0}=0$ by convention.

(ii) $X=\left\{X_{t}, 0 \leqslant t<\zeta_{\omega}, P\right\}$ is a Markov process on $S$ with transition function $p_{t}$ and initial distribution concentrated at $\{a\}$.

Proof. We shall employ the following notations:

$$
F\left(X ; t ; \alpha_{1}, f_{1}, \ldots, \alpha_{n}, f_{n}\right)=\int_{t<t_{1}<\cdots<t_{n}<\zeta_{\omega}} \ldots \prod_{k=1}^{n}\left\{\mathrm{e}^{-\alpha_{k}\left(t_{k}-t_{k-1}\right)} f_{k}\left(X_{t_{k}}\right)\right\} \mathrm{d} t_{1} \cdots \mathrm{d} t_{n},
$$

and, for $w \in W_{a}$,

$$
F\left(w ; t ; \alpha_{1}, f_{1}, \ldots, \alpha_{n}, f_{n}\right)=\int_{t<t_{1}<\cdots<t_{n}<\zeta(w)} \ldots \prod_{k=1}^{n}\left\{\mathrm{e}^{-\alpha_{k}\left(t_{k}-t_{k-1}\right)} f_{k}\left(w\left(t_{k}\right)\right)\right\} \mathrm{d} t_{1} \cdots \mathrm{d} t_{n} .
$$

(i) The left-hand side of (4.43) will be denoted by $G\left(\alpha_{1}, f_{1}, \ldots, \alpha_{n}, f_{n}\right)$, namely,

$$
E\left\{F\left(X ; 0 ; \alpha_{1}, f_{1}, \ldots, \alpha_{n}, f_{n}\right)\right\}=G\left(\alpha_{1}, f_{1}, \ldots, \alpha_{n}, f_{n}\right) .
$$

For $0<s<T$, we denote by $I(s)$ the expression

$$
\int_{J(s-)<t_{1}<J(s)} \mathrm{e}^{-\alpha_{1} t_{1}} f_{1}\left(X_{t_{1}}\right)\left\{\int_{t_{1}<t_{2}<\cdots<t_{n}<\zeta \omega} \ldots \prod_{k=2}^{n}\left(\mathrm{e}^{-\alpha_{k}\left(t_{k}-t_{k-1}\right)} f_{k}\left(X_{t_{k}}\right)\right) \mathrm{d} t_{2} \cdots \mathrm{d} t_{n}\right\} \mathrm{d} t_{1} .
$$

Then

$$
F\left(X ; 0 ; \alpha_{1}, f_{1}, \ldots, \alpha_{n}, f_{n}\right)=\sum_{0<s<T} I(s)+F\left(X ; J(T-) ; \alpha_{1}, f_{1}, \ldots, \alpha_{n}, f_{n}\right) .
$$

Further, if we put for $1 \leqslant m \leqslant n$

$$
\begin{aligned}
I_{m}(s)= & \int_{J(s-)<t_{1}<\cdots<t_{m}<J(s)} \cdots \prod_{k=1}\left\{\mathrm{e}^{-\alpha_{k}\left(t_{k}-t_{k-1}\right)} f_{k}\left(X_{t_{k}}\right)\right\} \mathrm{d} t_{1} \cdots \mathrm{d} t_{m} \\
& \times \int_{J(s)<t_{m+1}<\cdots<t_{n}<\zeta_{\omega}} \cdots \prod_{\ell=m+1}\left\{\mathrm{e}^{-\alpha_{\ell}\left(t_{\ell}-t_{\ell-1}\right)} f_{\ell}\left(X_{t_{\ell}}\right)\right\} \mathrm{d} t_{m+1} \cdots \mathrm{d} t_{n},
\end{aligned}
$$

then

$$
I(s)=\sum_{m=1}^{n} I_{m}(s)
$$

Moreover, each $I_{m}(s)$ can be written as

$$
I_{m}(s)=F_{m}(s) G_{m}(s)
$$

with 


$$
\begin{aligned}
& F_{m}(s)=\int_{J(s-)<t_{1}<\cdots<t_{m}<J(s)} \cdots \prod_{k=1}^{m}\left\{\mathrm{e}^{-\alpha_{k}\left(t_{k}-t_{k-1}\right)} f_{k}\left(X_{t_{k}}\right)\right\} \mathrm{e}^{-\alpha_{m+1}\left(J(s)-t_{m}\right)} \mathrm{d} t_{1} \cdots \mathrm{d} t_{m}, \\
& G_{m}(s)=\int_{J(s)<t_{m+1}<\cdots<t_{n}<\zeta_{\omega}} \cdots \mathrm{e}^{-\alpha_{m+1}\left(t_{m+1}-J(s)\right)} \prod_{\ell=m+2}^{n}\left\{\mathrm{e}^{-\alpha_{\ell}\left(t_{\ell}-t_{\ell-1}\right)} f_{\ell}\left(X_{t_{\ell}}\right)\right\} \mathrm{d} t_{m+1} \cdots \mathrm{d} t_{n} .
\end{aligned}
$$

Therefore

$$
F\left(X ; 0 ; \alpha_{1}, f_{1}, \ldots, \alpha_{n}, f_{n}\right)=\sum_{0<s<T} \sum_{m=1}^{n} F_{m}(s) G_{m}(s)+F\left(X ; J(T-) ; \alpha_{1}, f_{1}, \ldots, \alpha_{n}, f_{n}\right) .
$$

Next, let us put (with the convention that $\alpha_{n+1}=0$ )

$$
\begin{aligned}
F & \left(w ; \alpha_{1}, f_{1}, \ldots, \alpha_{m}, f_{m} ; \alpha_{m+1}\right) \\
& =\int_{0<t_{1}<\cdots<t_{m}<\zeta(w)} \cdots \prod_{k=1}^{m}\left\{\mathrm{e}^{-\alpha_{k}\left(t_{k}-t_{k-1}\right)} f_{k}\left(w\left(t_{k}\right)\right)\right\} \mathrm{e}^{-\alpha_{m+1}\left(\zeta(w)-t_{m}\right)} \mathrm{d} t_{1} \cdots \mathrm{d} t_{m},
\end{aligned}
$$

so that

$$
F_{m}(s)=\mathrm{e}^{-\alpha_{1} J(s-)} F\left(\mathbf{p}_{s}^{+} ; \alpha_{1}, f_{1}, \ldots, \alpha_{m}, f_{m} ; \alpha_{m+1}\right) .
$$

We furthermore put $Y_{t}=X_{J(s)+t}$ so that

$$
G_{m}(s)=\int_{0<t_{m+1}<\cdots<t_{n}<\zeta_{\omega}-J(s)} \prod_{\ell=m+1}^{n}\left\{\mathrm{e}^{-\alpha_{\ell}\left(t_{\ell}-t_{\ell-1}\right)} f_{\ell}\left(X_{t_{\ell}}\right)\right\} \mathrm{d} t_{m+1} \cdots \mathrm{d} t_{n},
$$

where we set $t_{m}=0$.

For $\mathbf{p}=\left\{\mathbf{p}_{t}, t>0\right\}$, we may use the following notations:

$$
\begin{aligned}
& G\left(\mathbf{p} ; \alpha_{m+1}, f_{m+1}, \ldots, \alpha_{n}, f_{n}\right) \\
& \quad=\int_{0<t_{m+1}<\cdots<t_{n}<\zeta_{\omega}} \cdots \prod_{\ell=m+1}^{n}\left\{\mathrm{e}^{-\alpha_{\ell}\left(t_{\ell}-t_{\ell-1}\right)} f_{\ell}\left(X_{t_{\ell}}\right)\right\} \mathrm{d} t_{m+1} \cdots \mathrm{d} t_{n}
\end{aligned}
$$

(with the convention that $t_{m}=0$ ), and

$$
\theta_{s} \mathbf{p}=\left\{\mathbf{p}_{s+t}, t>0\right\} .
$$

$\theta_{s} \mathbf{p}$ then has the same distribution as $\mathbf{p}$ and independent of $\left\{\mathbf{p}_{t}, 0<t<s\right\}$. Since $Y_{t}$ is constructed from $\theta_{s} \mathbf{p}$ in the same way as $X_{t}$ is from $\mathbf{p},(4.48)$ can be rewritten as

$$
G_{m}(s)=G\left(\theta_{s} \mathbf{p} ; \alpha_{m+1}, f_{m+1}, \ldots, \alpha_{n}, f_{n}\right),
$$

which is identical in law to

$$
G\left(\mathbf{p} ; \alpha_{m+1}, f_{m+1}, \ldots, \alpha_{n}, f_{n}\right)
$$

for each fixed $s>0$. Further

$$
F\left(X ; J(T-) ; \alpha_{1}, f_{1}, \ldots, \alpha_{n}, f_{n}\right)=\mathrm{e}^{-\alpha_{1} J(T-)} F\left(\mathbf{p}_{T}^{-} ; 0 ; \alpha_{1}, f_{1}, \ldots, \alpha_{n}, f_{n}\right) .
$$

Combining (4.45), (4.47), (4.51) and (4.52), we arrive at 


$$
\begin{aligned}
& F\left(X ; 0 ; \alpha_{1}, f_{1}, \ldots, \alpha_{n}, f_{n}\right) \\
& \qquad \sum_{0<s<T} \sum_{m=1}^{n} \mathrm{e}^{-\alpha_{1} J(s-)} F\left(\mathbf{p}_{s}^{+} ; \alpha_{1}, f_{1}, \ldots, \alpha_{m}, f_{m} ; \alpha_{m+1}\right) \\
& \quad \times G\left(\theta_{s} \mathbf{p} ; \alpha_{m+1}, f_{m+1}, \ldots, \alpha_{n}, f_{n}\right)+\mathrm{e}^{-\alpha_{1} J(T-)} F\left(\mathbf{p}_{T}^{-} ; 0 ; \alpha_{1}, f_{1}, \ldots, \alpha_{n}, f_{n}\right) .
\end{aligned}
$$

Here we compute the expectations of the random variables appearing in the last formula.

$$
\mathbf{n}^{+}\left\{F\left(w ; \alpha_{1}, f_{1}, \ldots, \alpha_{m}, f_{m} ; \alpha_{m+1}\right)\right\}=\hat{\mu}_{\alpha_{1}}\left(f_{1} G_{\alpha_{2}}^{0} f_{2} \cdots G_{\alpha_{m-1}}^{0} f_{m-1} G_{\alpha_{m}}^{0} f_{m} u_{\alpha_{m+1}}\right) .
$$

When $m=n$, the last factor $u_{\alpha_{n+1}}$ in the above expression is understood to be $u_{0}=\varphi$. In fact, the left-hand side equals

$$
\begin{aligned}
& \mathbf{n}\left\{\int_{0<t_{1}<\cdots<t_{m}<\zeta(w)} \ldots \prod_{k=1}^{m}\left(\mathrm{e}^{-\alpha_{k}\left(t_{k}-t_{k-1}\right)} f_{k}\left(w\left(t_{k}\right)\right)\right) \mathrm{e}^{-\alpha_{m+1}\left(\zeta(w)-t_{m}\right)} \mathrm{d} t_{1} \cdots \mathrm{d} t_{m} ; W_{a}^{+}\right\} \\
& =\int_{0<t_{1}<\cdots<t_{m}<\infty} \cdots \int_{k=1} \mathbf{n}\left\{\prod_{k}^{m}\left(\mathrm{e}^{-\alpha_{k}\left(t_{k}-t_{k-1}\right)} f_{k}\left(w\left(t_{k}\right)\right)\right) u_{\alpha_{m+1}}\left(w\left(t_{m}\right)\right) ; \zeta>t_{m}\right\},
\end{aligned}
$$

which can be seen to coincide with the right-hand side of (4.54) by (4.4).

We further have for any constant time $s>0$,

$$
E\left\{G\left(\theta_{s} \mathbf{p} ; \alpha_{m+1}, f_{m+1}, \ldots, \alpha_{n}, f_{n}\right)\right\}=G\left(\alpha_{m+1}, f_{m+1}, \ldots, \alpha_{n}, f_{n}\right) .
$$

On the other hand, we have in view of $\S 4.2$

$$
\begin{aligned}
& E\left\{F\left(\mathbf{p}_{T}^{-} ; 0 ; \alpha_{1}, f_{1}, \ldots, \alpha_{n}, f_{n}\right)\right\}=L\left(m_{0}, \psi\right)^{-1} \mathbf{n}^{-}\left\{F\left(w ; 0 ; \alpha_{1}, f_{1}, \ldots, \alpha_{n}, f_{n}\right)\right\} \\
& =L\left(m_{0}, \psi\right)^{-1} \hat{\mu}_{\alpha_{1}}\left(f_{1} G_{\alpha_{2}}^{0} f_{2} \cdots G_{\alpha_{n-1}}^{0} f_{n-1} G_{\alpha_{n}}^{0} f_{n} \psi\right), \\
& E\left\{\int_{0}^{T} \mathrm{e}^{-\alpha_{1} J(s)} \mathrm{d} s\right\}=\frac{1}{\alpha\left(u_{\alpha_{1}}, \varphi\right)+L\left(m_{0}, \psi\right)}, \\
& E\left\{\mathrm{e}^{-\alpha_{1} J(T-)}\right\}=\frac{L\left(m_{0}, \psi\right)}{\alpha\left(u_{\alpha_{1}}, \varphi\right)+L\left(m_{0}, \psi\right)} .
\end{aligned}
$$

We can now get from (4.53) that

$$
\begin{aligned}
G\left(\alpha_{1}, f_{1}, \ldots, \alpha_{n}, f_{n}\right)=E\left\{F\left(X ; 0 ; \alpha_{1}, f_{1}, \ldots, \alpha_{n}, f_{n}\right)\right\} \\
=\sum_{m=1}^{n} E\left\{\int_{0}^{T} \mathrm{e}^{-\alpha_{1} J(s)} \mathrm{d} s\right\} \mathbf{n}^{+}\left\{F\left(w ; \alpha_{1}, f_{1}, \ldots, \alpha_{m} ; \alpha_{m+1}\right)\right\} \\
\quad \times G\left(\alpha_{m+1}, f_{m+1}, \ldots, \alpha_{n}, f_{n}\right)+E\left\{\mathrm{e}^{-\alpha_{1} J(T-)}\right\} E\left\{F\left(\mathbf{p}^{-} ; 0 ; \alpha_{1}, f_{1}, \ldots, \alpha_{n}, f_{n}\right)\right\} \\
=\sum_{m=1}^{n-1} \frac{1}{\alpha\left(u_{\alpha}, \varphi\right)+L\left(m_{0}, \psi\right)} \hat{\mu}_{\alpha_{1}}\left(f_{1} G_{\alpha_{2}}^{0} f_{2} \cdots G_{\alpha_{m-1}}^{0} f_{m-1} G_{\alpha_{m}}^{0} f_{m} u_{\alpha_{m+1}}\right) \\
\quad \times G\left(\alpha_{m+1}, f_{m+1}, \ldots, \alpha_{n}, f_{n}\right)+\frac{1}{\alpha\left(u_{\alpha}, \varphi\right)+L\left(m_{0}, \psi\right)} \hat{\mu}_{\alpha_{1}}\left(f_{1} G_{\alpha_{2}}^{0} f_{2} \cdots G_{\alpha_{n-1}}^{0} f_{n-1} G_{\alpha_{n}}^{0} f_{n} \varphi\right) \\
\quad+\frac{L\left(m_{0}, \psi\right)}{\alpha\left(u_{\alpha}, \varphi\right)+L\left(m_{0}, \psi\right)} L\left(m_{0}, \psi\right)^{-1} \hat{\mu}_{\alpha_{1}}\left(f_{1} G_{\alpha_{2}}^{0} f_{2} \cdots G_{\alpha_{n-1}}^{0} f_{n-1} G_{\alpha_{n}}^{0} f_{n} \psi\right)
\end{aligned}
$$




$$
=\frac{1}{\alpha\left(u_{\alpha}, \varphi\right)+L\left(m_{0}, \psi\right)} \sum_{m=1}^{n} \hat{\mu}_{\alpha_{1}}\left(f_{1} G_{\alpha_{2}}^{0} f_{2} \cdots G_{\alpha_{m-1}}^{0} f_{m-1} G_{\alpha_{m}}^{0} f_{m} u_{\alpha_{m+1}}\right) G\left(\alpha_{m+1}, f_{m+1}, \ldots, \alpha_{n}, f_{n}\right) .
$$

In the above and in what follows, we use the convention that

$$
u_{\alpha_{m+1}}=G\left(\alpha_{m+1}, f_{m+1}, \ldots, \alpha_{n}, f_{n}\right)=1
$$

for $m=n$. This combined with (4.1) and (4.30) eventually leads us to

$$
\begin{aligned}
& G\left(\alpha_{1}, f_{1}, \ldots, \alpha_{n}, f_{n}\right) \\
& \quad=\sum_{m=1}^{n} G_{\alpha_{1}}\left(f_{1} G_{\alpha_{2}}^{0} f_{2} \cdots G_{\alpha_{m-1}}^{0} f_{m-1} G_{\alpha_{m}}^{0} f_{m} u_{\alpha_{m+1}}\right)(a) G\left(\alpha_{m+1}, f_{m+1}, \ldots, \alpha_{n}, f_{n}\right) .
\end{aligned}
$$

Based on this formula, we shall prove the desired identity (4.43), namely,

$$
G\left(\alpha_{1}, f_{1}, \ldots, \alpha_{n}, f_{n}\right)=G_{\alpha_{1}} f_{1} G_{\alpha_{2}} f_{2} \cdots G_{\alpha_{n}} f_{n}(a)
$$

by induction in $n$.

(1) When $n=1,(4.59)$ is just (4.30).

(2) Suppose (4.59) holds up to $n-1$. Then

$$
G\left(\alpha_{m+1}, f_{m+1}, \ldots, \alpha_{n}, f_{n}\right)=\left(G_{\alpha_{m+1}} f_{m+1} \cdots G_{\alpha_{n}} f_{n}\right)(a),
$$

and (4.58) can be written as

$$
\begin{aligned}
G\left(\alpha_{1}, f_{1}, \ldots, \alpha_{n}, f_{n}\right)= & \sum_{m=1}^{n} G_{\alpha_{1}}\left(f_{1} G_{\alpha_{2}}^{0} f_{2} \cdots G_{\alpha_{m-1}}^{0} f_{m-1} G_{\alpha_{m}}^{0} f_{m} u_{\alpha_{m+1}}\right)(a) \\
& \times\left(G_{\alpha_{m+1}} f_{m+1} \cdots G_{\alpha_{n}} f_{n}\right)(a) .
\end{aligned}
$$

Let us rewrite the right-hand side of (4.59) by applying the formula (4.31) to the operation $G_{\alpha_{2}}$ in getting

$$
\left(G_{\alpha_{1}} f_{1} G_{\alpha_{2}} f_{2} \cdots G_{\alpha_{n}} f_{n}\right)(a)=\left(G_{\alpha_{1}} f_{1} G_{\alpha_{2}}^{0} f_{2} G_{\alpha_{3}} f_{3} \cdots G_{\alpha_{n}} f_{n}\right)(a)+\left(G_{\alpha_{1}} f_{1} u_{\alpha_{2}}\right)(a)\left(G_{\alpha_{2}} f_{2} \cdots G_{\alpha_{n}} f_{n}\right)(a) .
$$

Apply the same procedure to the operation $G_{\alpha_{3}}$ to see that the right-hand side of (4.59) equals

$$
\begin{aligned}
& \left(G_{\alpha_{1}} f_{1} G_{\alpha_{2}}^{0} f_{2} G_{\alpha_{3}}^{0} f_{3} G_{\alpha_{4}} f_{4} \cdots G_{\alpha_{n}} f_{n}\right)(a)+\left(G_{\alpha_{1}} f_{1} G_{\alpha_{2}}^{0} f_{2} u_{\alpha_{3}}\right)(a)\left(G_{\alpha_{3}} f_{3} \cdots G_{\alpha_{n}} f_{n}\right)(a) \\
& \quad+\left(G_{\alpha_{1}} f_{1} u_{\alpha_{2}}\right)(a)\left(G_{\alpha_{2}} f_{2} \cdots G_{\alpha_{n}} f_{n}\right)(a) .
\end{aligned}
$$

Repeating the same procedures, we finally find that the right-hand side of (4.59) coincides with the right-hand side of (4.60) as was to be proved.

(ii) For $t_{1}>0, \ldots, t_{n}>0$, let

$$
\begin{aligned}
& F\left(t_{1}, \ldots, t_{n}\right)=E\left\{\prod_{k=1}^{n} f_{k}\left(X_{t_{1}+\cdots+t_{k}}\right) ; \zeta_{\omega}>t_{1}+\cdots+t_{n}\right\}, \\
& G\left(t_{1}, \ldots, t_{n}\right)=\left(p_{t_{1}} f_{1} p_{t_{2}} f_{2} \cdots p_{t_{n}} f_{n}\right)(a) .
\end{aligned}
$$

(4.43) is then equivalent to

$$
\int_{0}^{\infty} \cdots \int_{0}^{\infty} \mathrm{e}^{-\alpha_{1} t_{1}-\cdots-\alpha_{n} t_{n}} F\left(t_{1}, \ldots, t_{n}\right) \mathrm{d} t_{1} \cdots \mathrm{d} t_{n}=\int_{0}^{\infty} \cdots \int_{0}^{\infty} \mathrm{e}^{-\alpha_{1} t_{1}-\cdots-\alpha_{n} t_{n}} G\left(t_{1}, \ldots, t_{n}\right) \mathrm{d} t_{1} \cdots \mathrm{d} t_{n} .
$$


Clearly $F\left(t_{1}, \ldots, t_{n}\right)$ is right continuous. Further, by virtue of Lemma 4.11 , we can easily see that $G\left(t_{1}, \ldots, t_{n}\right)$ is separately right continuous. Consequently, (4.61) implies

$$
F\left(t_{1}, \ldots, t_{n}\right)=G\left(t_{1}, \ldots, t_{n}\right)
$$

the desired Markov property of $X$.

We add a lemma saying that the point $a$ is regular for itself with respect to $\left(X_{t}, P\right)$.

\section{Lemma 4.12.}

(i) $P\left(\eta_{a}=0\right)=1$, where $\eta_{a}=\inf \left\{t>0: X_{t}=a\right\}$.

(ii) $\mathbf{n}^{+}\left(W_{a}\right)=\infty$.

Proof. (i) In view of the proof of Proposition 4.3, $\lim _{t \downarrow} u_{1}\left(X_{t}\right)=1$. Hence, if we put $\eta_{a, \epsilon}=\inf \left\{t>\epsilon: X_{t}=a\right\}$, then owing to the Markov property

$$
E\left(\mathrm{e}^{-\eta_{a}}\right)=\lim _{\epsilon \downarrow 0} E\left(\mathrm{e}^{-\eta_{a, \epsilon}}\right)=\lim _{\epsilon \downarrow 0} E\left(\mathrm{e}^{-\epsilon} u_{1}\left(X_{\epsilon}\right) ; \zeta_{\omega}>\epsilon\right)=1 .
$$

(ii) By the construction of $X_{t}$, the point $a$ is evidently instantaneous in the sense that

$$
P\left(\tau_{a}=0\right)=1, \quad \text { where } \tau_{a}=\inf \left\{t>0: X_{t} \in S_{0}\right\} .
$$

Hence (i) holds if and only if the domain $D_{\mathbf{p}^{+}}$of the Poisson point process $\mathbf{p}^{+}$accumulates at $0 P$-a.s., which is also equivalent to (ii) (cf. $[15, \S 4])$.

\subsection{A symmetric extension $\widetilde{X}$ of $X^{0}$}

In $\S 4.1$, we have started with an $m$-symmetric diffusion

$$
X^{0}=\left\{X_{t}^{0}, 0 \leqslant t<\zeta^{0}, P_{x}^{0}, x \in S_{0}\right\}
$$

on $S_{0}$, where $P_{x}^{0}, x \in S_{0}$, are probability measures on a certain sample space, say $\Omega^{0}$.

In $\S 4.2$, we have constructed a continuous process

$$
X=\left\{X_{t}, 0 \leqslant t<\zeta_{\omega}, P\right\}
$$

on $S$ by piecing together the excursions, where $P$ is a probability measure on another sample space $\Omega$ to define the excursion valued Poisson point processes.

For convenience, we assume that $\Omega^{0}$ contains an extra point $\omega^{a}$ with $P_{x}^{0}\left(\left\{\omega^{a}\right\}\right)=0, x \in S_{0}$, and we set $P_{a}^{0}=\delta_{\omega^{a}}, \omega^{a}$ representing a path taking value $a$ at any time.

We now let

$$
\widetilde{\Omega}=\Omega^{0} \times \Omega, \quad \widetilde{P}_{x}=P_{x}^{0} \times P, \quad x \in S .
$$

For $\widetilde{\omega}=\left(\omega^{0}, \omega\right) \in \widetilde{\Omega}$, let us define $\widetilde{X}_{t}=\widetilde{X}_{t}(\widetilde{\omega})$ as follows:

(1) When $\omega^{0} \in \Omega^{0} \backslash\left\{\omega^{a}\right\}$,

$$
\tilde{X}_{t}(\widetilde{\omega})= \begin{cases}X_{t}^{0}\left(\omega^{0}\right), & 0 \leqslant t<\zeta^{0}\left(\omega^{0}\right) \leqslant \sigma_{a}\left(\omega^{0}\right) \leqslant \infty, \\ X_{t-\sigma_{a}\left(\omega^{0}\right)}(\omega), & \sigma_{a}\left(\omega^{0}\right) \leqslant t<\sigma_{a}\left(\omega^{0}\right)+\zeta_{\omega}, \text { if } \sigma_{a}\left(\omega^{0}\right)<\infty .\end{cases}
$$

(2) When $\omega^{0}=\omega^{a}$,

$$
\widetilde{X}_{t}(\widetilde{\omega})=X_{t}(\omega), \quad 0 \leqslant t<\zeta_{\omega} .
$$


The life time $\tilde{\zeta}$ of $\tilde{X}_{t}$ is defined by

$$
\tilde{\zeta}= \begin{cases}\zeta^{0} & \text { if } \sigma_{a}\left(\omega^{0}\right)=\infty \\ \sigma_{a}\left(\omega^{0}\right)+\zeta_{\omega} & \text { if } \sigma_{a}\left(\omega^{0}\right)<\infty\end{cases}
$$

Lemma 4.13. $\widetilde{X}=\left\{\widetilde{X}_{t}, 0 \leqslant t<\tilde{\zeta}, \widetilde{P}_{x}, x \in S\right\}$ is a Markov process on $S$ with transition function $\left\{p_{t}\right\}$ defined by (4.35) and (4.36).

Proof. This is an easy consequence of the Markov property of $\left(X_{t}^{0}, P_{x}^{0}\right)$ and the Markov property of $\left(X_{t}, P\right)$ proved in Proposition 4.4. To see this, we put, for any $0<s_{1}<s_{2}<\cdots<s_{n}, f_{1}, f_{2}, \ldots, f_{n} \in \mathcal{B}(S)$,

$$
I_{k}=\widetilde{E}_{x}\left(f_{1}\left(\widetilde{X}_{s_{1}}\right) \cdots f_{k-1}\left(\widetilde{X}_{s_{k-1}}\right) f_{k}\left(\widetilde{X}_{s_{k}}\right) \cdots f_{n}\left(\widetilde{X}_{s_{n}}\right) ; s_{k-1}<\sigma_{a} \leqslant s_{k}\right),
$$

for $1 \leqslant k \leqslant n$ with $s_{0}=0$, and

$$
J=\widetilde{E}_{x}\left(f_{1}\left(\widetilde{X}_{s_{1}}\right) \cdots f_{n}\left(\widetilde{X}_{s_{n}}\right) ; s_{n}<\sigma_{a}\right)
$$

Using the definition of $\tilde{X}$, Proposition 4.4, the Markov property of $X^{0}$ and (4.36) successively, we are led to

$$
\begin{aligned}
& I_{k}=E_{x}^{0}\left(f_{1}\left(X_{s_{1}}^{0}\right) \cdots f_{k-1}\left(X_{s_{k-1}}^{0}\right) E\left(f_{k}\left(X_{s_{k}-\sigma_{a}}\right) \cdots f_{n}\left(X_{s_{n}-\sigma_{a}}\right)\right) ; s_{k-1}<\sigma_{a} \leqslant s_{k}\right) \\
& =E_{x}^{0}\left(f_{1}\left(X_{s_{1}}^{0}\right) \cdots f_{k-1}\left(X_{s_{k-1}}^{0}\right) p_{s_{k}-\sigma_{a}}\left(f_{k} p_{s_{k+1}-s_{k}} f_{k+1} \cdots p_{s_{n}-s_{n-1}} f_{n}\right)(a) ; s_{k-1}<\sigma_{a} \leqslant s_{k}\right) \\
& =E_{x}^{0}\left\{f_{1}\left(X_{s_{1}}^{0}\right) \cdots f_{k-1}\left(X_{s_{k-1}}^{0}\right)\right. \\
& \left.\times E_{X_{s_{k-1}}^{0}}^{0}\left(p_{s_{k}-s_{k-1}-\sigma_{a}}\left(f_{k} p_{s_{k+1}-s_{k}} f_{k+1} \cdots p_{s_{n}-s_{n-1}} f_{n}\right) ; \sigma_{a} \leqslant s_{k}-s_{k-1}\right) ; s_{k-1}<\sigma_{a} \leqslant s_{k}\right\} \\
& =E_{x}^{0}\left(f_{1}\left(X_{s_{1}}^{0}\right) \cdots f_{k-1}\left(X_{s_{k-1}}^{0}\right)\right. \\
& \left.\times\left(p_{s_{k}-s_{k-1}}-p_{s_{k}-s_{k-1}}^{0}\right)\left(f_{k} p_{s_{k+1}-s_{k}} f_{k+1} \cdots p_{s_{n}-s_{n-1}} f_{n}\right)\left(X_{s_{k-1}}^{0}\right) ; s_{k-1}<\sigma_{a} \leqslant s_{k}\right) .
\end{aligned}
$$

By the Markov property of $X^{0}$, we thus get

$$
\begin{aligned}
I_{k}= & p_{s_{1}}^{0} f_{1} \cdots p_{s_{k-1}-s_{k-2}}^{0} f_{k-1} p_{s_{k}-s_{k-1}} f_{k} p_{s_{k+1}-s_{k}} f_{k+1} \cdots p_{s_{n}-s_{n-1}} f_{n}(x) \\
& -p_{s_{1}}^{0} f_{1} \cdots p_{s_{k-1}-s_{k-2}}^{0} f_{k-1} p_{s_{k}-s_{k-1}}^{0} f_{k} p_{s_{k+1}-s_{k}} f_{k+1} \cdots p_{s_{n}-s_{n-1}} f_{n}(x) .
\end{aligned}
$$

Clearly we also have

$$
J=E_{x}^{0}\left(f_{1}\left(X_{s_{1}}^{0}\right) \cdots f_{n}\left(X_{s_{n}}^{0}\right) ; s_{n}<\sigma_{a}\right)=p_{s_{1}}^{0} f_{1} \cdots p_{s_{n}-s_{n-1}}^{0} f_{n} .
$$

Hence we arrive at

$$
\widetilde{E}_{x}\left(f_{1}\left(\tilde{X}_{s_{1}}\right) f_{2}\left(\tilde{X}_{s_{2}}\right) \cdots f_{n}\left(\tilde{X}_{s_{n}}\right)\right)=\sum_{k=1}^{n} I_{k}+J=p_{s_{1}} f_{1} p_{s_{2}-s_{1}} f_{2} \cdots p_{s_{n}-s_{n-1}} f_{n}(x),
$$

the desired Markov property of $\tilde{X}$.

We now state main theorems of the present paper. In this section, we have started with an $m$-symmetric diffusion $X^{0}$ on $S_{0}$ satisfying conditions A.1-A.4 and constructed a Markov process $\widetilde{X}$ on $S$. The resolvent $\left\{G_{\alpha}\right\}_{\alpha>0}$ of the Markov process $\widetilde{X}$ is defined by

$$
G_{\alpha} f(x)=\widetilde{E}_{x}\left(\int_{0}^{\infty} \mathrm{e}^{-\alpha t} f\left(\widetilde{X}_{t}\right) \mathrm{d} t\right), \quad f \in \mathcal{B}(S) .
$$

The resolvent of $X^{0}$ was denoted by $G_{\alpha}^{0}$. 
Theorem 4.1. The process $\widetilde{X}$ enjoys the following properties:

(1) $\tilde{X}$ is an $m$-symmetric diffusion process on $S$. It admits no killing inside $S$ and is a Hunt process on $S$ in the sense that

$$
\tilde{X}_{\tilde{\zeta}(\widetilde{\omega})-}(\widetilde{\omega})=\Delta \quad \text { if } \tilde{\zeta}(\widetilde{\omega})<\infty .
$$

(2) $X^{0}$ is identical in law with the process obtained from $\widetilde{X}$ by killing upon the hitting time $\sigma_{a}$ of the point a.

Further the resolvent of $\widetilde{X}$ admits the next expression for $f \in \mathcal{B}(S)$ :

$$
\begin{aligned}
G_{\alpha} f(x) & =G_{\alpha}^{0} f(x)+u_{\alpha}(x) \frac{\left(u_{\alpha}, f\right)}{\alpha\left(u_{\alpha}, \varphi\right)+L\left(m_{0}, \psi\right)}, \quad x \in S_{0}, \\
G_{\alpha} f(a) & =\frac{\left(u_{\alpha}, f\right)}{\alpha\left(u_{\alpha}, \varphi\right)+L\left(m_{0}, \psi\right)},
\end{aligned}
$$

where $L\left(m_{0}, \psi\right)$ is the energy functional of the $X^{0}$-excessive measure $m_{0}=\varphi \cdot m$ and the $X^{0}$-excessive function $\psi=1-\varphi$.

Proof of Theorem 4.1. By Lemma 4.6, (4.37) and Lemma 4.13, we see that $\widetilde{X}$ is a Markov process on $S$ with the $m$-symmetric resolvent (4.67), (4.68).

On account of A.1, we may assume that

$$
X_{t}^{0}\left(\omega^{0}\right) \text { is continuous in } t \in\left[0, \zeta^{0}\left(\omega^{0}\right)\right) \text { and } X_{\zeta^{0}\left(\omega^{0}\right)-}\left(\omega^{0}\right)=a \cup \Delta
$$

for every $\omega^{0} \in \Omega^{0}$. We have already chosen $\Omega$ in a way that

$$
X_{t}(\omega) \text { is continuous in } t \in\left[0, \zeta_{\omega}\right) \text { and } X_{0}(\omega)=a .
$$

Hence the path $\widetilde{X} .(\widetilde{\omega})$ defined by $(4.63)-(4.65)$ is continuous on $[0, \tilde{\zeta})$.

Consider a function $u=G_{\alpha} f$ on $S$ for $f \in C_{b}(S)$. By the assumptions A.2, A.3 and the expression (4.67), (4.68), $u\left(X_{t}^{0}\left(\omega^{0}\right)\right)$ is then continuous in $t \in\left[0, \sigma_{a}\right)$ for any $\omega^{0} \in \Omega^{0}$. By the proof of Proposition $4.3, u\left(X_{t}(\omega)\right)$ is continuous in $t \in\left[0, \zeta_{\omega}\right)$ for any $\omega \in \Omega$. Hence $u\left(\widetilde{X}_{t}(\widetilde{\omega})\right)$ is right continuous in $t \in[0, \tilde{\zeta}(\widetilde{\omega}))$ for any $\widetilde{\omega} \in \widetilde{\Omega}$. (In view of (4.33), we even know that $u\left(\widetilde{X}_{t}\right)$ is continuous in $t \in[0, \tilde{\zeta}) \widetilde{P}_{x}$-a.s. for any $x \in S$.) Therefore we can conclude that $\widetilde{X}$ is a strong Markov process with continuous sample paths, namely, a diffusion process on $S$ (cf. [2]). Clearly $\widetilde{X}$ is of no killing inside $S$ and a Hunt process on $S$. The property (2) is also evident from the construction of $\widetilde{X}$.

Remark 4.1. A prime reason for us to impose a regularity condition A.4 on the given process $X^{0}$ on $S_{0}$ is in that it implies an important property in Lemma 4.3 of the excursion law $\mathbf{n}$ of (4.4), which is essential in deriving the continuity near the point $a$ of the process $X$ constructed in $\S 4.2$.

Given a standard process $\widetilde{X}$ on $S$ for which the point $a$ is recurrent, K. Itô [15] associated with $\widetilde{X}$ a Poisson point process $\mathbf{p}$ of excursions in the manner of $\S 3.1$ and gave a list of necessary conditions for the characteristic measure n of $\mathbf{p}$ should obey. Conversely T.S. Salisbury [24,25] constructed a right process on $S$ for which $a$ is recurrent by means of $X^{0}$ and an excursion law $\mathbf{n}$ satisfying Itô's conditions being strengthened by adding the property as in Lemma 4.3 and some others.

Remark 4.2. By invoking the work of P.A. Meyer [20] on the absorbed Poisson point process and by adopting a similar argument to $\S 4.2$, we can show that Theorem 3.1 of $\S 3.1$ remains true without assuming condition B.3 on the recurrence of the point $\{a\}$. 
In this general case, the right continuous inverse $S(s)$ of the local time $L(t)$ at $\{a\}$ of the given process $X$ on $S$ is defined for $s \geqslant L(\infty)$ as $S(s)=\infty$, and we see from Lemma 2.3 and by letting $\alpha \downarrow 0$ in $(2.21)$ that $L(\infty)$ has an exponential distribution with mean $L\left(m_{0}, \psi\right)^{-1}$.

Let

$$
\begin{aligned}
& D_{\mathbf{p}}=\{s: S(s)-S(s-)>0\} \\
& \mathbf{p}_{s}(t)=X_{S(s-)+t}, s \in D_{\mathbf{p}}, 0 \leqslant t<S(s)-S(s-) .
\end{aligned}
$$

Then $D_{\mathbf{p}} \subset(0, L(\infty)], L(\infty) \in D_{\mathbf{p}}$ and $\left\{\mathbf{p}_{s}, s>0\right\}$ is a point process with values in the space $W_{a}$ defined by (4.13) instead of (3.6). Moreover, if we define the spaces $W_{a}^{+}, W_{a}^{-}$by (4.14), (4.15) respectively, then

$$
\mathbf{p}_{s} \in W_{a}^{+} \quad \text { for } s \in D_{\mathbf{p}} \cap(0, L(\infty)), \quad \mathbf{p}_{L(\infty)} \in W_{a}^{-} .
$$

By Theorem 5 of Meyer [20], $\left\{\mathbf{p}_{s}, s>0\right\}$ is an absorbed Poisson point process. More precisely, on a certain probability space $(\widetilde{\Omega}, \widetilde{P})$, there is a Poisson point process $\left\{\tilde{\mathbf{p}}_{s}, s>0\right\}$ on $W_{a}$ with domain $D_{\tilde{\mathbf{p}}}$ and with the following properties.

(a) Let $\tilde{\zeta}=\inf \left\{s>0: \tilde{\mathbf{p}}_{s} \in W_{a}^{-}\right\}$and consider the stopped point process $\{\overline{\mathbf{p}}, s>0\}$ :

$$
\overline{\mathbf{p}}_{s}=\tilde{\mathbf{p}}_{s} \quad \text { for } s \in D_{\overline{\mathbf{p}}}=D_{\tilde{\mathbf{p}}} \cap(0, \tilde{\zeta}] .
$$

Then the point process $\left\{\mathbf{p}_{s}, s>0\right\}$ and $\left\{\overline{\mathbf{p}}_{s}, s>0\right\}$ are equivalent in law.

(b) Let $\mathbf{n}$ be the characteristic measure of $\left\{\tilde{\mathbf{p}}_{s}, s>0\right\}$. Then $\{w(t), \mathbf{n}\}$ is Markovian with respect to the transition function $p_{t}^{0}$ of $X^{0}$. Let $\left\{v_{t}\right\}$ be the entrance law associated with $\mathbf{n}$. Then $v_{t}$ is a finite measure for each $t>0$ and $\int_{0}^{\infty} \mathrm{e}^{-t} v_{t} \mathrm{~d} t$ has a total mass not greater than 1 .

We now prove that Theorem 3.1 remains valid for this $\left\{v_{t}\right\}$ and for the entrance law $\left\{\mu_{t}\right\}$ specified by the Eq. (2.22).

Take a bounded Borel function $f$ on $S$ and define $\hat{f}_{\alpha}(w), w \in W_{a}, \alpha>0$, as in the proof of Proposition 4.2. We have, almost surely with respect to $P_{a}$,

$$
\begin{aligned}
\int_{0}^{\zeta} \mathrm{e}^{-\alpha t} f\left(X_{t}\right) \mathrm{d} t & =\sum_{s<L(\infty)} \int_{S(s-)}^{S(s)} \mathrm{e}^{-\alpha t} f\left(X_{t}\right) \mathrm{d} t+\int_{S(L(\infty)-)}^{\infty} \mathrm{e}^{-\alpha t} f\left(X_{t}\right) \mathrm{d} t \\
& =\sum_{s<L(\infty)} \mathrm{e}^{-\alpha S(s-)} \hat{f}_{\alpha}\left(\mathbf{p}_{s}\right)+\mathrm{e}^{-\alpha S(L(\infty)-)} \hat{f}_{\alpha}\left(\mathbf{p}_{L(\infty)}\right),
\end{aligned}
$$

which is equivalent in law to

$$
\sum_{s<\tilde{\zeta}} \mathrm{e}^{-\alpha \widetilde{S}(s-)} \hat{f}_{\alpha}\left(\tilde{\mathbf{p}}_{s}^{+}\right)+\mathrm{e}^{-\widetilde{S}(\tilde{\zeta}-)} \hat{f}_{\alpha}\left(\tilde{\mathbf{p}}_{\tilde{\zeta}}\right),
$$

where $\left\{\tilde{\mathbf{p}}_{s}^{+}, s>0\right\}$ is a Poisson point process defined by $\tilde{\mathbf{p}}_{s}^{+}=\tilde{\mathbf{p}}_{s}$ for $s \in D_{\tilde{\mathbf{p}}^{+}}=D_{\tilde{\mathbf{p}}} \cap\left\{s: \tilde{\mathbf{p}}_{s} \in W_{a}^{+}\right\}$and $\widetilde{S}(s)=$ $\sum_{r \leqslant s} \zeta\left(\tilde{\mathbf{p}}_{r}^{+}\right)$. The characteristic measure of $\left\{\tilde{\mathbf{p}}_{s}^{+}, s>0\right\}$ is the restriction $\mathbf{n}^{+}$of $\mathbf{n}$ on $W_{a}^{+}$. In the same way as in the proof of Lemma 4.5, we can prove that

$$
\widetilde{E}\left(\mathrm{e}^{-\alpha \widetilde{S}(s)}\right)=\exp \left(-\alpha \hat{v}_{\alpha}(\varphi)\right), \quad \hat{v}_{\alpha}=\int_{0}^{\infty} \mathrm{e}^{-\alpha t} v_{t} \mathrm{~d} t .
$$

Now the value $G_{\alpha} f(a)$ equals the expectation of the random variable (4.69) with respect to $\widetilde{P}$, which can be evaluated by taking into account of the following facts. 
(i) The three objects $\left\{\tilde{\mathbf{p}}_{s}^{+}, s>0\right\}, \tilde{\zeta}$ and $\tilde{\mathbf{p}}_{\tilde{\zeta}}$ are independent.

(ii) $\tilde{\zeta}$ has an exponential distribution with mean $L\left(m_{0}, \psi\right)^{-1}$.

(iii) The law of $\tilde{\mathbf{p}}_{\tilde{\zeta}}$ is $L\left(m_{0}, \psi\right)^{-1} \mathbf{n}^{-}$where $\mathbf{n}^{-}$is the restriction of $\mathbf{n}$ on $W_{a}^{-}$.

Indeed, exactly the same computation as in the proof of Proposition 4.2 leads us to

$$
G_{\alpha} f(a)=\frac{\hat{v}_{\alpha}(f)}{\alpha \hat{v}_{\alpha}(\varphi)+L\left(m_{0}, \psi\right)},
$$

which combined with (2.15) and Lemma 2.2(ii) yields

$$
\frac{\hat{v}_{\alpha}(f)}{\alpha \hat{v}_{\alpha}(\varphi)+L\left(m_{0}, \psi\right)}=\frac{\hat{\mu}_{\alpha}(f)}{\alpha \hat{\mu}_{\alpha}(\varphi)+L\left(m_{0}, \psi\right)} .
$$

Therefore for each $\alpha>0$ there is a constant $c_{\alpha}$ such that $\hat{v}_{\alpha}=c_{\alpha} \hat{\mu}_{\alpha}$. Inserting this into the above equation, we easily obtain $c_{\alpha}=1$ and so $v_{t}=\mu_{t}, t>0$.

\section{Uniqueness of the symmetric extension and expression of its Dirichlet form}

In the preceding section, we have started with an $m$-symmetric diffusion $X^{0}$ on $S_{0}$ satisfying conditions A.1-A.4, and constructed a process $\widetilde{X}$ on $S$ satisfying properties (1), (2) stated in Theorem 4.1. Let us call a process on $S$ satisfying conditions (1), (2) a symmetric extension of $X^{0}$. In this section, we are concerned with the uniqueness of a symmetric extension of $X^{0}$ and explicit expression of its Dirichlet form on $L^{2}(S ; m)$. We aim at proving the following:

Theorem 5.1. Assume that an $m$-symmetric diffusion $X^{0}$ on $S_{0}$ satisfies conditions A.1, A.2. Let $\widehat{X}$ be a symmetric extension of $X^{0}$ and $(\mathcal{E}, \mathcal{F})$ be the Dirichlet form on $L^{2}(S ; m)$ of $\widehat{X}$.

(i) $\widehat{X}$ admits the resolvent identical with (4.67), (4.68).

(ii) $(\mathcal{E}, \mathcal{F})$ admits the expression

$$
\begin{aligned}
& \mathcal{F}_{e}=\left\{w=u_{0}+c \varphi: u_{0} \in \mathcal{F}_{o, e}, c \text { constant }\right\}, \quad \mathcal{F}=\mathcal{F}_{e} \cap L^{2}(S ; m), \\
& \mathcal{E}(w, w)=\mathcal{E}\left(u_{0}, u_{0}\right)+c^{2} \mathcal{E}(\varphi, \varphi), \quad \mathcal{E}(\varphi, \varphi)=L\left(m_{0}, \psi\right),
\end{aligned}
$$

where $\left(\mathcal{F}_{0, e}, \mathcal{E}\right)$ is the extended Dirichlet space of $X^{0}$ and $L\left(m_{0}, \psi\right)$ is the energy functional of $m_{0}=\varphi \cdot m$ and $\psi$ with respect to $X^{0}$.

(iii) $X^{0}$ satisfies A.3 automatically: $u_{\alpha} \in L^{1}(S ; m), \alpha>0$.

(iv) $\widehat{P}_{a}\left(\sigma_{a}=0, \tau_{a}=0\right)=1$ where $\sigma_{a}=\inf \left\{t>0: X_{t}=a\right\}, \tau_{a}=\inf \left\{t>0: X_{t} \in S_{0}\right\}$.

(v) $(\mathcal{E}, \mathcal{F})$ is irreducible.

Corollary 5.1. Under the conditions A.1, A.2 for an m-symmetric diffusion $X^{0}$ on $S_{0}$, the symmetric extension of $X^{0}$ is unique in law.

Corollary 5.1 follows from Theorem 5.1(i). We prepare a lemma before the proof of Theorem 5.1.

Assume that $X=\left(X_{t}, P_{x}\right)$ is an $m$-symmetric Hunt process on $S$ and $(\mathcal{E}, \mathcal{F})$ is the associated Dirichlet form on $L^{2}(S ; m)$. No regularity for the Dirichlet form $(\mathcal{E}, \mathcal{F})$ is assumed in advance.

In accordance with [19], we set for a closed set $F \subset S$,

$$
\mathcal{F}_{F}=\{u \in \mathcal{F}: u=0 m \text {-a.e. on } S \backslash F\},
$$


and call an increasing family $\left\{F_{n}\right\}$ of closed subsets of $S$ an $\mathcal{E}$-nest if the space $\bigcup_{n=1}^{\infty} \mathcal{F}_{F_{n}}$ is $\mathcal{E}_{1}$-dense in $\mathcal{F}$. A set $N$ is called $\mathcal{E}$-exceptional if $N \subset \bigcap_{n=1}^{\infty} F_{n}^{c}$ for some $\mathcal{E}$-nest $\left\{F_{n}\right\}$. On the other hand, we call a set $N \subset S$ an $X$-exceptional set if there exists a Borel set $B_{1} \supset B$ with

$$
P_{m}\left(\sigma_{B_{1}}<\infty\right)=0 .
$$

A nearly Borel set $N \subset S$ is called $X$-properly exceptional if $m(N)=0$ and $S \backslash N$ is $X$-invariant in the sense that

$$
P_{x}\left(X_{t} \in S_{\Delta} \backslash N \text { or } X_{t-} \in S_{\Delta} \backslash N \exists t \geqslant 0\right)=1, \quad \forall x \in S \backslash N .
$$

\section{Lemma 5.1.}

(i) The following properties of a set $N \subset S$ are equivalent each other:

$\alpha . N$ is $\mathcal{E}$-exceptional.

$\beta . N$ is $X$-exceptional.

$\gamma . N$ is contained in an $X$-properly exceptional Borel set.

(ii) If $\left\{F_{n}\right\}$ is an $\mathcal{E}$-nest, then

$$
P_{x}\left(\lim _{n \rightarrow \infty} \sigma_{S \backslash F_{n}} \geqslant \zeta\right)=1 \text { q.e., }
$$

where q.e. means 'except on a set $N \subset S$ satisfying one of the properties in (i)'.

(iii) $(\mathcal{E}, \mathcal{F})$ is a quasi-regular Dirichlet form on $L^{2}(S ; m)$ in the sense of $[19, \S$ IV 3].

Proof. (i) The equivalences $\alpha \Leftrightarrow \beta$ and $\beta \Leftrightarrow \gamma$ were proved in [19, Theorem 5.29] and in [9, Theorem 4.1.1] respectively.

(ii) Put $\sigma=\lim _{n \rightarrow \infty} \sigma_{S \backslash F_{n}}$. On account of [19, Theorem 2.11, Theorem 5.4], we have for a strictly positive bounded $m$-integrable function $f$ on $S$,

$$
E_{x}\left(\int_{\sigma \wedge \zeta}^{\zeta} \mathrm{e}^{-s} f\left(X_{S}\right) \mathrm{d} s\right)=0 \quad m-\text { a.e. } x \in S
$$

Since the function of $x$ on the left-hand side of the above equation is $X$-excessive, it is finely continuous on $S$ and hence the above equation holds q.e. by [9, Lemma 4.1.5].

(iii) Since $(\mathcal{E}, \mathcal{F})$ is associated with a Hunt process $X$, it must be quasi-regular by virtue of [19, Theorem 5.1].

Proof of Theorem 5.1. Since $\widehat{X}$ is not only a diffusion process but also a Hunt process on $S$, the Dirichlet form $(\mathcal{E}, \mathcal{F})$ of $\widehat{X}$ is quasi-regular by the above lemma.

Consequently we can invoke [3, Theorem 3.7] to find a regular Dirichlet space $\left(S^{\prime}, m^{\prime}, \mathcal{F}^{\prime}, \mathcal{E}^{\prime}\right)$ related to the quasi-regular Dirichlet space $(S, m, \mathcal{F}, \mathcal{E})$ by a quasi-homeomorphism $q$ : there exist an $\mathcal{E}$-nest $\left\{F_{n}\right\}$ on $S$ and an $\mathcal{E}^{\prime}$-nest $\left\{F_{n}^{\prime}\right\}$ on $S^{\prime}$ such that $q$ is a one to one mapping from $S_{1}=\bigcup_{n=1}^{\infty} F_{n}$ onto $S_{1}^{\prime}=\bigcup_{n=1}^{\infty} F_{n}^{\prime}$ and its restriction on each $F_{n}$ is homeomorphic to $F_{n}^{\prime}$. Further, $m^{\prime}$ is the image measure of $m$ by $q$ and the space $\left(\mathcal{F}^{\prime}, \mathcal{E}^{\prime}\right)$ is also the image of $(\mathcal{F}, \mathcal{E})$ by $q$. Thus, if we put $(\Phi u)\left(x^{\prime}\right)=u\left(q^{-1}\left(x^{\prime}\right)\right), x^{\prime} \in S_{1}^{\prime}$, then

$$
\int_{S^{\prime}}(\Phi u) \mathrm{d} m^{\prime}=\int_{S} u \mathrm{~d} m, \quad \forall u \geqslant 0 ; \quad \mathcal{F}^{\prime}=\Phi(\mathcal{F}), \quad \mathcal{E}^{\prime}(\Phi u, \Phi v)=\mathcal{E}(u, v), \quad u, v \in \mathcal{F} .
$$

We note that $S \backslash S_{1}$ (resp. $\left.S^{\prime} \backslash S_{1}^{\prime}\right)$ is $\mathcal{E}$-(resp. $\mathcal{E}^{\prime}$-)exceptional and, when $N^{\prime}=q(N), N$ is $\mathcal{E}$-exceptional if and only if $N^{\prime}$ is $\mathcal{E}^{\prime}$-exceptional (cf. [3, Corollary 3.6]). 
For a Borel set $B \subset S$, we denote by $B_{\Delta}$ the subset $B \cup \Delta$ of $S_{\Delta}$ with induced topology. The above $q$ can then be extended to a homeomorphism between $\left(F_{n}\right)_{\Delta}$ and $\left(F_{n}^{\prime}\right)_{\Delta^{\prime}}$ for each $n$, where $\Delta^{\prime}$ denotes the point at infinity of $S^{\prime}$ (which is added as an isolated point when $S^{\prime}$ is compact).

We now apply Lemma 5.1 to the above $\mathcal{E}$-nest $\left\{F_{n}\right\}$ in finding an $\widehat{X}$-properly exceptional Borel set $\widehat{N} \subset S$ containing $S \backslash S_{1}$ such that (5.3) holds for any $x \in S \backslash \widehat{N} . q$ is then a one-to-one mapping between $S \backslash \widehat{N}$ and $S^{\prime} \backslash \widehat{N}^{\prime}$, where

$$
\widehat{N}^{\prime}=\left(S^{\prime} \backslash S_{1}^{\prime}\right) \cup q(S \cap \widehat{N}) .
$$

In view of condition A.2 for $X^{0}$, condition (2) for $\widehat{X}$ and the above observation, the one point set $\{a\}$ is not $\widehat{X}$-exceptional and consequently it is not $\mathcal{E}$-exceptional by virtue of Lemma 5.1 . Therefore $a$ must be located in $S \backslash \widehat{N}$ and furthermore

$$
\left\{a^{\prime}\right\} \text { is not } \mathcal{E}^{\prime} \text {-exceptional, }
$$

where $a^{\prime}=q(a) \subset S^{\prime} \backslash \widehat{N}^{\prime}$.

The restriction of $\widehat{X}$ to $S \backslash \widehat{N}$ is a diffusion with no killing inside $S \backslash \widehat{N}$ and we denote it again by

$$
\widehat{X}=\left(\Omega, \mathcal{F}_{t}, \widehat{X}_{t}, \hat{\zeta}, \widehat{P}_{x}\right) .
$$

Let us transfer $\widehat{X}$ to a process

$$
\widehat{X}^{\prime}=\left(\Omega, \mathcal{F}_{t}, \widehat{X}_{t}^{\prime}, \hat{\zeta}^{\prime}, \widehat{P}_{x}^{\prime}\right)
$$

on $S^{\prime} \backslash \widehat{N}^{\prime}$ by the mapping $q$ :

$$
\begin{aligned}
& \widehat{X}_{t}^{\prime}(\omega)=q\left(\widehat{X}_{t}\right)(\omega), \quad \hat{\zeta}^{\prime}(\omega)=\hat{\zeta}(\omega), \quad \omega \in \Omega, t \geqslant 0, \\
& \widehat{P}_{x}^{\prime}(\Lambda)=\widehat{P}_{q^{-1}{ }_{x}}(\Lambda), \quad x \in S^{\prime} \backslash \widehat{N}^{\prime}, \Lambda \in \mathcal{F}_{\infty} .
\end{aligned}
$$

We may extend the state space of $\widehat{X}^{\prime}$ to $S^{\prime}$ by making each point of $\widehat{N}^{\prime}$ trap. It is then easy to see that $\widehat{X}^{\prime}$ is a diffusion process on $S^{\prime}$ with no killing inside $S^{\prime}$ in the sense that

$$
\widehat{P}_{x}^{\prime}\left(\hat{\zeta}^{\prime}<\infty, \widehat{X}_{\hat{\zeta}^{\prime}-}^{\prime}=\Delta\right)=\widehat{P}_{x}^{\prime}\left(\hat{\zeta}^{\prime}<\infty\right) \text {. }
$$

Further $\widehat{X}^{\prime}$ is associated with the Dirichlet form $\left(\mathcal{E}^{\prime}, \mathcal{F}^{\prime}\right)$ which is regular. Since $\widehat{X}^{\prime}$ is a diffusion without killing inside $S^{\prime},\left(\mathcal{E}^{\prime}, \mathcal{F}^{\prime}\right)$ must be strongly local (cf. [9, Theorem 4.5.3]). By (5.5) and Lemma 5.1, we see that the one point set $\left\{a^{\prime}\right\}$ is not $\widehat{X}^{\prime}$-exceptional and consequently it has a positive capacity with respect to $\left(\mathcal{E}^{\prime}, \mathcal{F}^{\prime}\right)$ in virtue of [9, Theorem 4.2.1].

Therefore $\left(\mathcal{E}^{\prime}, \mathcal{F}^{\prime}\right)$ and $\widehat{X}^{\prime}$ fit the setting of $\S 2$ and they satisfy all the properties stated in Theorem 2.1 of $\S 2$. In particular, we have the next expressions of the resolvent and $\left(\mathcal{E}^{\prime}, \mathcal{F}^{\prime}\right)$ of $\widehat{X}^{\prime}$ in terms of the part $\widehat{X}^{\prime}, 0$ of $\widehat{X}^{\prime}$ on $S_{0}^{\prime}=S^{\prime} \backslash\left\{a^{\prime}\right\}$ : if we denote the transition function and the resolvent of $\widehat{X}^{\prime}\left(\right.$ resp. $\widehat{X}^{\prime, 0}$ ) by $p_{t}^{\prime}, G_{\alpha}^{\prime}\left(\right.$ resp. $\left.p_{t}^{\prime, 0}, G_{\alpha}^{\prime, 0}\right)$, then

$$
\begin{aligned}
& G_{\alpha}^{\prime} g\left(a^{\prime}\right)=\frac{\left(u_{\alpha}^{\prime}, g\right)_{m^{\prime}}}{\alpha\left(u_{\alpha}^{\prime}, \varphi^{\prime}\right)_{m^{\prime}}+L^{\prime}\left(m_{0}^{\prime}, \psi^{\prime}\right)}, \\
& \mathcal{E}^{\prime}\left(\varphi^{\prime}, \varphi^{\prime}\right)=L^{\prime}\left(m_{0}^{\prime}, \psi^{\prime}\right),
\end{aligned}
$$

where $\varphi^{\prime}$ (resp. $u_{\alpha}^{\prime}$ ) is the hitting (resp. $\alpha$-order hitting) probability of $\left\{a^{\prime}\right\}$ of the process $\widehat{X}^{\prime}, \psi^{\prime}=1-\varphi^{\prime}$ and

$$
L^{\prime}\left(m_{0}^{\prime}, \psi^{\prime}\right)=\lim _{t \downarrow 0} \frac{1}{t}\left(\varphi^{\prime}-p_{t}^{\prime, 0} \varphi^{\prime}, \psi^{\prime}\right)_{m^{\prime}}
$$

Notice that the part $\left(\mathcal{E}^{\prime}, \mathcal{F}_{0}^{\prime}\right)$ of $\left(\mathcal{E}^{\prime}, \mathcal{F}^{\prime}\right)$ on $S_{0}^{\prime}$ is associated with $\widehat{X}^{\prime}, 0$ which can be sent from $X^{0}$ on $S_{0}$ by the mapping $q$ in the same way as above on account of the property (2) of $\widehat{X}$. Hence we have for $x \in S^{\prime} \backslash \widehat{N}^{\prime}$ 


$$
\begin{aligned}
& \Phi\left(G_{\alpha} f\right)(x)=G_{\alpha}^{\prime}(\Phi f)(x), \quad \Phi\left(G_{\alpha}^{0} f\right)(x)=G_{\alpha}^{\prime, 0}(\Phi f)(x), \quad \Phi\left(p_{t}^{0} f\right)(x)=p_{t}^{\prime, 0}(\Phi f)(x), \\
& \Phi(\varphi)(x)=\varphi^{\prime}(x), \quad \Phi\left(u_{\alpha}\right)(x)=u_{\alpha}^{\prime}(x) .
\end{aligned}
$$

(5.4)-(5.9) and (5.10) now imply $L^{\prime}\left(m_{0}^{\prime}, \psi^{\prime}\right)=L\left(m_{0}, \psi\right)$ and furthermore

$$
\mathcal{E}(\varphi, \varphi)=L\left(m_{0}, \psi\right), \quad G_{\alpha} f(a)=\frac{\left(u_{\alpha}, f\right)}{\alpha\left(u_{\alpha}, \varphi\right)+L\left(m_{0}, \psi\right)}
$$

We have obtained the expression (4.68) of the resolvent $G_{\alpha}$ of $\widehat{X}$. It then satisfies (4.67) for all $x \in S_{0}$ because of the property (2) of $\widehat{X}$. We can also readily get the assertions (ii) and (iii) of Theorem 5.1 using (5.4) and (5.10). As for (iv), we have obviously

$$
\widehat{P}_{a}\left(\sigma_{a}=0, \tau_{a}=0\right)=\widehat{P}_{a^{\prime}}^{\prime}\left(\sigma_{a^{\prime}}=0, \tau_{a^{\prime}}=0\right),
$$

and the right-hand side equals 1 by virtue of Theorem 2.1. From the expression (4.67) of the resolvent of $\widehat{X}$, we have

$$
\left(I_{A}, G_{\alpha} I_{B}\right)>0 \text { for any } A, B \in \mathcal{B}(S) \text { with } m(A)>0, m(B)>0 .
$$

This property is equivalent to the irreducibility of the Dirichlet form $(\mathcal{E}, \mathcal{F})$ proving $(\mathrm{v})$.

Remark 5.1. For the symmetric extension $\widetilde{X}$ of $X^{0}$ constructed in $\S 4$, not only the expression (4.67), (4.68) of its resolvent but also the property (iv) in Theorem 5.1 have been directly proved in Lemma 4.12.

\section{Examples}

Example 6.1. Let $X$ be the Brownian motion on $\mathbb{R}, X^{0}$ be the absorbed Brownian motion on $\mathbb{R} \backslash\{0\}$ and $m$ be the Lebesgue measure $\mathrm{d} x$ on $\mathbb{R}$. Then $X$ is the unique $m$-symmetric extension of $X^{0}$ (in the sense that $X$ satisfies conditions (1), (2) of Theorem 4.1) in accordance with Corollary 5.1.

Let $L(t)$ be the local time of $X$ at 0 and $Z$ be an independent exponential random variable with mean $\delta^{-1}$. The process $X_{\delta}$ obtained from $X$ killed upon the first time that $L(t) \geqslant Z$ is a diffusion process extending $X^{0}$ but not a symmetric extension of $X^{0}$ in the present sense because it violates the above condition (1).

For $\gamma>0$, let $X^{\gamma}$ be the process on $\mathbb{R}$ obtained from $X$ by a time change with respect to the inverse of its additive functional $t+\gamma L(t) . X^{\gamma}$ is then a diffusion on $\mathbb{R}$ with a canonical scale $2 \mathrm{~d} x$ and the speed measure $m(\mathrm{~d} x)=\mathrm{d} x+\gamma \delta_{0}(\mathrm{~d} x) . X^{\gamma}$ extends $X^{0}$ but violates our assumption that $m(\{0\})=0$.

The resolvents and Dirichlet forms of $X_{\delta}, X^{\gamma}$ have been exhibited in Remark 2.2.

Example 6.2. Let $D$ be a bounded open set in $\mathbb{R}^{d}(d \geqslant 1)$, and $L^{2}(D)$ be the $L^{2}$-space based on the Lebesgue measure on $D$. Denote by $H_{0}^{1}(D)$ the closure of $C_{0}^{1}(D)$ in the Sobolev space

$$
H^{1}(D)=\left\{u \in L^{2}(D): \frac{\partial u}{\partial x_{i}} \in L^{2}(D), 1 \leqslant i \leqslant n\right\}
$$

and put

$$
\mathbf{D}(u, v)=\int_{D} \nabla u \cdot \nabla v(x) \mathrm{d} x, \quad u, v \in H_{0}^{1}(D) .
$$

Then $\left(\frac{1}{2} \mathbf{D}, H_{0}^{1}(D)\right)$ is a strongly local Dirichlet form on $L^{2}(D)$ satisfying the Poincaré inequality (3.13). The associated symmetric diffusion $X^{0}=\left(X_{t}^{0}, 0 \leqslant t<\zeta^{0}, P_{x}^{0}\right)$ on $D$ is the absorbing Brownian motion. 
Let $D^{*}=D \cup\{a\}$ be the one point compactification of $D$. Regarding $D$ as a subspace of $D^{*}$, we have then

$$
\begin{aligned}
& \varphi(x)=P_{x}^{0}\left(\zeta^{0}<\infty, X_{\zeta^{0}-}^{0}=a\right)=1, \quad \psi(x)=1-\varphi(x)=0, \quad \forall x \in D, \\
& u_{\alpha}(x)=E_{x}^{0}\left(\mathrm{e}^{-\alpha \zeta^{0}} ; X_{\zeta^{0}-}^{0}=a\right) \text { is continuous in } x \in D(\alpha>0) .
\end{aligned}
$$

Obviously $u_{\alpha} \in L^{1}(D)$. Hence conditions A.1-A.4 are satisfied by $X^{0}$ and we can construct a diffusion $\widetilde{X}$ on $D^{*}$ as in $\S 4$. By virtue of Theorem 4.1, the resolvent of $\widetilde{X}$ is expressed as

$$
G_{\alpha} f(x)=G_{\alpha}^{0} f(x)+u_{\alpha}(x) \frac{\left(u_{\alpha}, f\right)}{\alpha\left(u_{\alpha}, 1\right)}, \quad x \in D, \quad G_{\alpha} f(a)=\frac{\left(u_{\alpha}, f\right)}{\alpha\left(u_{\alpha}, 1\right)},
$$

and in particular, $\widetilde{X}$ is conservative.

$L^{2}\left(D^{*}\right)$ denotes the $L^{2}$-space based on the 0-extension of the Lebesgue measure on $D$ to $D^{*}$. By virtue of Theorems 4.1 and 5.1, $\widetilde{X}$ is symmetric with respect to this measure and its Dirichlet form $(\mathcal{E}, \mathcal{F})$ on $L^{2}\left(D^{*}\right)$ is describable as

$$
\begin{aligned}
& \mathcal{F}=H_{0}^{1}(D)+\text { constant functions on } D^{*}, \\
& \mathcal{E}\left(w_{1}, w_{2}\right)=\frac{1}{2} \mathbf{D}\left(f_{1}, f_{2}\right), \quad w_{i}=f_{i}+c_{i}, f_{i} \in H_{0}^{1}(D), c_{i} \text { constant }, i=1,2 .
\end{aligned}
$$

On account of Theorem 3.2 and a related observation in $\$ 3.1$, this is a regular, strongly local and irreducible recurrent Dirichlet form. This Dirichlet form first appeared in [8].

The entrance law $\left\{\mu_{t}\right\}_{t>0}$ governing the characteristic measure of the excursion valued Poisson point process attached to $\widetilde{X}$ is given by

$$
\mu_{t}(B) \mathrm{d} t=\int_{B} P_{x}^{0}\left(\zeta^{0} \in \mathrm{d} t\right) \mathrm{d} x, \quad B \in \mathcal{B}(D),
$$

in view of (3.9). Let $D=\bigcup_{i} D_{i}$ be the decomposition of the open set $D$ into connected components. The above identity tells us that the sample path of $\widetilde{X}$ entering from the point $a$ is distributed among $\left\{D_{i}\right\}$ proportionally to their volumes and enters in $D_{i}$ according to the restriction of $\mu_{t}$ to $D_{i}$. As was observed in $\S 3.1, \widetilde{X}$ is irreducible recurrent.

According to (2.24), the Lévy measure of the inverse local time of $\tilde{X}$ at the point $a$ is given by $-\mathrm{d} \mu_{t}(D)$.

Example 6.3. We consider a finite number of disjoint rays $\ell_{i}, i=1, \ldots, N$, on $\mathbb{R}^{2}$ merging at a point $a \in \mathbb{R}^{2}$. Each ray $\ell_{i}$ is homeomorphic to the open half line $(0, \infty)$ and the point $a$ is the boundary of each ray at 0 -side. We put

$$
S_{0}=\sum_{i=1}^{N} \ell_{i}, \quad S=S_{0}+a .
$$

$S$ is endowed with the induced topology as a subset of $\mathbb{R}^{2}$.

Let $m$ be a positive Radon measure on $S_{0}$ with $\operatorname{Supp}[m]=S_{0} . m$ is extended to $S$ by setting $m(\{a\})=0$. The restriction of $m$ to $\ell_{i}$ is denoted by $m_{i}$. For any function $g$ on $S_{0}$, its restriction to $\ell_{i}$ will be denoted by $g_{i}$. We consider a diffusion process $X^{0}=\left\{X_{t}^{0}, \zeta^{0}, P_{x}^{0}\right\}$ on $S_{0}$ such that its restriction $X^{0, i}$ to each open half line $\ell_{i} \sim(0, \infty)$ is the absorbing diffusion governed by the speed measure $m_{i}$ and a canonical scale, say $s_{i}$.

We notice that $X^{0}$ satisfies A.2, A.3 if and only if 0 is a regular boundary in Feller's sense for each diffusion $X^{0, i}$ on $\ell_{i}, 1 \leqslant i \leqslant N$. Indeed, A.2 holds if and only if 0 is exit (in the terminology used by [16]). If 0 is additionally non-entrance, then $m_{i}((0,1))=\infty$ and A.3 is not satisfied. If 0 is regular, then $m_{i}((0,1))<\infty$ and $u_{\alpha, i}$ is $m_{i}$ integrable on $(0,1)$, while $u_{\alpha, i}$ is always $m_{i}$-integrable on $[1, \infty)$ (cf. [16, p 130]).

Thus we assume that 0 is regular for every $X^{0, i}$ so that A.1-A.3 are satisfied by $X^{0}$. A.4 is also clearly satisfied. $m$ is finite on any compact neighbourhood of $a$. 
Therefore, a diffusion $\widetilde{X}$ on $S$ can be constructed as in $\S 4$ and it is a unique $m$-symmetric extension of $X^{0}$ with no killing inside $S$ according to Theorem 5.1. The resolvent of $\widetilde{X}$ has the expression

$$
G_{\alpha} f(a)=\frac{\sum_{i}\left(u_{\alpha, i}, f_{i}\right)_{m_{i}}}{\alpha \sum_{i}\left(u_{\alpha, i}, \varphi_{i}\right)_{m_{i}}+\sum_{i} L\left(\varphi_{i} \cdot m_{i}, \psi_{i}\right)} .
$$

The Dirichlet form $(\mathcal{E}, \mathcal{F})$ of $\tilde{X}$ on $L^{2}(S ; m)$ is regular, strongly local, irreducible and can be described as follows:

$$
\begin{aligned}
& \mathcal{F}_{e}=\left\{w=u_{0}+c \varphi: u_{0} \in \mathcal{F}_{0, e}, c \text { constant }\right\} \\
& \mathcal{E}(w, w)=\mathcal{E}\left(u_{0}, u_{0}\right)+c^{2} \mathcal{E}(\varphi, \varphi) \\
& \mathcal{E}(\varphi, \varphi)=\sum_{i} L\left(\varphi_{i} \cdot m_{i}, \psi_{i}\right)
\end{aligned}
$$

where

$$
\begin{aligned}
\mathcal{F}_{0, e}=\{u: & u_{i} \text { is absolutely continuous with respect to } s_{i}, \\
& \left.\int_{0}^{\infty}\left(\frac{\mathrm{d} u_{i}}{\mathrm{~d} s_{i}}\right)^{2} \mathrm{~d} s_{i}<\infty, u_{i}(0)=0, u_{i}(\infty)=0, \text { whenever } \infty \text { is regular, } 1 \leqslant i \leqslant n\right\}, \\
\mathcal{E}(u, u)= & \sum_{i} \int_{0}^{\infty}\left(\frac{\mathrm{d} u_{i}}{\mathrm{~d} s_{i}}\right)^{2} \mathrm{~d} s_{i}, \quad u \in \mathcal{F}_{0, e} .
\end{aligned}
$$

Related Dirichlet forms and diffusions first appeared in [13].

The entrance law from $a$ is describable as

$$
\mu_{t}(f) \mathrm{d} t=\sum_{i} P_{f_{i} \cdot m_{i}}^{0, i}\left(\zeta^{0, i} \in \mathrm{d} t, X_{\zeta^{0, i}-}^{0, i}=0\right)
$$

We have a freedom of choice of the entrance law (6.6) in the following sense. Choose any positive numbers $\left\{p_{1}, \ldots, p_{N}\right\}$ and observe that the absorbed diffusion $X^{0}$ on $S_{0}$ is unchanged if we replace $m_{i}, s_{i}, 1 \leqslant i \leqslant N$, by

$$
\widehat{m}_{i}=p_{i} \cdot m_{i}, \quad \hat{s}_{i}=p_{i}^{-1} \cdot s_{i}, \quad 1 \leqslant i \leqslant N,
$$

respectively. Let $\widehat{m}$ be the measure on $S$ whose restriction to $\ell_{i}$ equals $\widehat{m}_{i}$ for each $i=1,2, \ldots, N$, with $\widehat{m}(\{a\})=0$. Then we can consider the $\widehat{m}$-symmetric extension $\widehat{X}$ of $X^{0}$ whose entrance law $\hat{\mu}$ from $a$ is given by (6.6) but with the replacement of $m_{i}$ by $\widehat{m}_{i}$ for $1 \leqslant i \leqslant N$.

Example 6.4. Let $G_{1}, G_{2}$ be open sets of $\mathbb{R}^{d}(d \geqslant 1)$, such that

$$
\bar{G}_{1} \subset G_{2}, \quad \bar{G}_{1} \text { is compact. }
$$

We let $S_{0}=G_{2} \backslash \bar{G}_{1}$. We consider the space $S=S_{0} \cup\{a\}$ equipped with the topology where a set $U$ containing $a$ is defined to be an open set if

$$
\left.U \backslash\{a\}=\text { open subset of } G_{2} \text { containing } \bar{G}_{1}\right\} \backslash \bar{G}_{1} .
$$

Let $X^{0}$ be the absorbing Brownian motion on $S_{0}$. Then conditions A.1-A.4 are satisfied by $X^{0}$. A.3 can be verified by a comparison with the Brownian motion on $\mathbb{R}^{d}$.

Let $m$ be the Lebesgue measure on $S_{0}$ extended to $S$ by $m(\{a\})=0$. Let $\widetilde{X}$ be the $m$-symmetric diffusion on $S$ as is constructed in $\S 4$. Then, by Theorem 5.1, its Dirichlet form $(\mathcal{E}, \mathcal{F})$ on $L^{2}(S ; m)$ is expressed as 


$$
\begin{aligned}
& \mathcal{F}=\mathcal{F}_{e} \cap L^{2}(S ; m), \quad \mathcal{F}_{e}=\left\{w=u_{0}+c \varphi: u_{0} \in H_{0, e}^{1}\left(S_{0}\right), c \text { constant }\right\}, \\
& \mathcal{E}(w, w)=\frac{1}{2} \mathbf{D}\left(u_{0}, u_{0}\right)+c^{2} L(\varphi \cdot m, \psi),
\end{aligned}
$$

where $H_{0, e}^{1}\left(S_{0}\right)$ denotes the extended Dirichlet space of $H_{0}^{1}\left(S_{0}\right)$.

$(\mathcal{E}, \mathcal{F})$ is a quasi-regular Dirichlet form on $L^{2}(S ; m)$ but may not be regular. It is a regular Dirichlet space if each point of $\partial G_{1}$ is a regular boundary point of $S_{0}$ with respect to the Dirichlet problem for $\left(\alpha-\frac{1}{2} \Delta\right)$ on $S_{0}$.

\section{Acknowledgements}

We are grateful to P.J. Fitzsimmons, Y. Le Jan and an anonymous referee for calling our attention to the literature closely related to the present subject. Thanks are also due to K. Burdzy for his helpful comment on Example 6.3.

\section{References}

[1] R.M. Blumenthal, Excursions of Markov Processes, Birkhäuser, Boston, 1992.

[2] R.M. Blumenthal, R.K. Getoor, Markov Processes and Potential Theory, Academic Press, New York, 1968.

[3] Z.-Q. Chen, Z.-M. Ma, M. Röckner, Quasi-homeomorphisms of Dirichlet forms, Nagoya Math. J. 136 (1994) 1-15.

[4] C. Dellacherie, P.A. Meyer, Probabilités et potentiel, Chap. XII, Hermann, Paris, 1987.

[5] C. Dellacherie, B. Maisonneuve, P.A. Meyer, Probabilités et potentiel, Chap. XVII-XXIV, Hermann, Paris, 1992.

[6] E.B. Dynkin, An application of flows to time shift and time reversal in stochastic processes, Trans. Amer. Math. Soc. 287 (1985) $613-619$.

[7] P.J. Fitzsimmons, On the excursions of Markov processes in classical duality, Probab. Theory Related Fields 75 (1987) $159-178$.

[8] M. Fukushima, On boundary conditions for multi-dimensional Brownian motions with symmetric resolvent densities, J. Math. Soc. Japan 21 (1969) 58-93.

[9] M. Fukushima, Y. Oshima, M. Takeda, Dirichlet Forms and Symmetric Markov Processes, de Gruyter, 1994.

[10] R.K. Getoor, Excursions of a Markov process, Ann. Probab. 7 (1979) 244-266.

[11] R.K. Getoor, Excessive Measures, Birkhäuser, 1990.

[12] R.K. Getoor, M.J. Sharpe, Excursions of dual processes, Adv. Math. 45 (1982) 259-309.

[13] N. Ikeda, S. Watanabe, The local structure of a class of diffusions and related problems, in: Lecture Notes in Math., vol. 330, 1973, pp. $124-159$.

[14] N. Ikeda, S. Watanabe, Stochastic Differential Equations and Diffusion Processes, North-Holland/Kodansha, 1981.

[15] K. Itô, Poisson point processes attached to Markov processes, in: Proc. Sixth Berkeley Symp. Math. Statist. Probab. III, 1970 , pp. $225-239$.

[16] K. Itô, H.P. McKean, Diffusion Processes and Their Sample Paths, Springer, 1970.

[17] D. Kim, On spectral gaps and exit time distributions for a non-smooth domain, Forum Math., in press.

[18] Y. Le Jan, Dual Markovian semigroups and processes, in: Functional Analysis in Markov Processes, Proceedings, Katata and Kyoto, 1981, in: Lecture Notes in Math., vol. 923, 1982, pp. 47-75.

[19] Z.M. Ma, M. Röckner, Introduction to the Theory of (Non-Symmetric) Dirichlet Forms, Springer-Verlag, 1992.

[20] P.A. Meyer, Processus de Poisson ponctuels, d'aprés K. Itô, in: Séminaire de Probab. V, in: Lecture Notes in Math., vol. 191, Springer, Berlin, 1971, pp. 177-190.

[21] P.A. Meyer, Note sur l'interprétation des measures d'équilibre, in: Séminaire de Probab. VII, in: Lecture Notes in Math., vol. 321, 1973, pp. 210-216.

[22] M. Nagasawa, Time reversion of Markov processes, Nagoya Math. J. 24 (1964) 177-204.

[23] L.C.G. Rogers, Itô excursion theory via resolvents, Z. Wahrsch. Verw. Gebiete 63 (1983) 237-255.

[24] T.S. Salisbury, On the Itô excursion process, Probab. Theory Related Fields 73 (1986) 319-350.

[25] T.S. Salisbury, Construction of right processes from excursions, Probab. Theory Related Fields 73 (1986) 351-367.

\section{Further reading}

[26] J. Mitro, Time reversal depending on local time, Stochastic Process. Appl. 18 (1984) 171-177. 